\title{
Tumor Necrosis Factor- $\alpha$ Initiates miRNA-mRNA Signaling Cascades in Obstruction-Induced Bladder Dysfunction
}

Ivonne Koeck, ${ }^{*}$ Ali Hashemi Gheinani, ${ }^{*}$ Ulrich Baumgartner, ${ }^{\dagger}$ Erik Vassella, ${ }^{\dagger}$ Rémy Bruggmann, ${ }^{\ddagger}$ Fiona C. Burkhard, ${ }^{* \S}$ and Katia Monastyrskaya ${ }^{\star}$

From the Urology Research Laboratory, ${ }^{*}$ Department for BioMedical Research, the Institute of Pathology, ${ }^{\dagger}$ and the Interfaculty Bioinformatics Unit, ${ }^{\ddagger}$ University of Bern, Bern; and the Department of Urology, ${ }^{\S}$ University Hospital, Bern, Switzerland

\author{
Accepted for publication \\ May 3, 2018. \\ Address correspondence to \\ Katia Monastyrskaya, Ph.D., \\ Urology Research Laboratory, \\ Department for BioMedical \\ Research, University of Bern, \\ Murtenstrasse 35, 3008 Bern, \\ Switzerland. E-mail: \\ monastyk@dbmr.unibe.ch.
}

\begin{abstract}
Bladder outlet obstruction (B00) and the ensuing clinical lower urinary tract dysfunction are common in elderly patients. BOO is accompanied by urodynamic changes in bladder function and leads to organ fibrosis and ultimately loss of contractility. Comprehensive transcriptome analysis of bladder samples from human patients with different urodynamically defined phenotypes of B00 revealed tumor necrosis factor (TNF)- $\alpha$ as the top upstream signaling pathway regulator. Herein, we validated next-generation sequencing and pathway analysis in cell-based models using bladder smooth muscle and urothelial cells exposed to TNF- $\alpha$. miRNA profiling and transcriptome analysis of TNF- $\alpha$-treated bladder smooth muscle cells revealed striking similarities with human B00. Using a comparative approach, TNF-specific and TNF-independent pathways were delineated in human biopsy specimens. Concomitant down-regulation of smooth muscle cell-specific miRNAs and smooth muscle markers after TNF- $\alpha$ treatment was in accordance with the loss of contractility in humans in advanced obstruction-induced bladder remodeling. The expression levels of four abundant TNF-regulated miRNAs were modulated; the compensatory up-regulation of miR-199a-5p reduced NF- $\kappa B$ signaling. Essential hubs of TNF- $\alpha$ signaling pathways mitogen-activated protein kinase kinase kinase (apoptosis signal-regulating kinase 1 ) and inhibitor of nuclear factor $\kappa$ B kinase subunit $\beta$ (I $\kappa B$ kinase $\beta$ ) were targeted by miR-199a-5p. miR199a-5p might be part of a negative feedback loop, reducing the impact of TNF, whereas its downregulation in acontractile bladders from B00 patients advances the disease. The compensatory upregulation of miR-199a-5p together with TNF- $\alpha$ inhibition may be therapeutically beneficial. (Am J Pathol 2018, 188: 1847-1864; https://doi.org/10.1016/j.ajpath.2018.05.008)
\end{abstract}

Bladder outlet obstruction (BOO) caused by benign prostatic hyperplasia is a frequent condition in older men and often leads to lower urinary tract dysfunction (LUTD). Approximately $25 \%$ of men aged between 40 and 49 years and $50 \%$ aged between 70 and 79 years experience moderate to severe LUTD. ${ }^{1}$ The functional outcomes of BOO are often reduced bladder compliance, changes in sensitivity, detrusor overactivity (DO), or detrusor underactivity (UA). Obstruction reduces or prevents the urinary flow from the bladder into the urethra, and intravesical pressure increase is required to void successfully. The chronically elevated pressure induces a magnitude of structural and functional changes in the bladder wall, resulting in bladder hypertrophy. ${ }^{2}$ The bladder wall thickness is increased proportionally to the severity of obstruction, ${ }^{3}$ and in men with benign prostatic hyperplasia, both parameters significantly correlate. ${ }^{4}$ The mechanosensitive smooth muscle cells (SMCs) in the bladder wall undergo modifications of gene expression and protein synthesis in response to mechanical stretch stress caused by BOO. Several transduction mechanisms trigger this process, ${ }^{5}$ and the inflammatory changes of

Supported by the Swiss National Science Foundation grant 320030_156161/1 (K.M.) and the Velux Foundation grant 895 (K.M.).

I.K. and A.H.G. contributed equally to this work.

Disclosures: None declared. 
the detrusor muscle gradually lead to hypertrophy and fibrosis. ${ }^{6}$ Changes in cell signaling and the consequent alterations of gene expression through miRNAs play a vital role in the progression of these dysfunctions. ${ }^{7}$ miRNAs are endogenous, noncoding, single-stranded RNAs that regulate gene expression and control adaptive and maladaptive organ remodeling processes. They have been shown to play an essential role in muscle cell differentiation. ${ }^{8}$ Furthermore, dysregulation of miRNAs contributes to many human diseases, ${ }^{9}$ including pain- and inflammation-related LUTD, which are accompanied by changes in miRNA expression. ${ }^{10}$ Some miRNAs have been implicated in other bladder pathologies ${ }^{10-15}$ and function, ${ }^{16}$ and there is evidence supporting their role in the regulation of urothelial permeability $^{11}$ and bladder contractility. ${ }^{13,17}$

Recently, we completed the quantitative transcriptome and miRNA profiling of different functional phenotypes of BOO. ${ }^{7}$ In bladder biopsy specimens from human patients with urodynamically defined phenotypes, an integrated analysis of miRNA and mRNA paired expression profiling was performed, and it was shown that the patients' miRNA and mRNA expression profiles correlated with urodynamic findings. Furthermore, we identified combinations of three mRNAs (NRXN3, BMP7, and UPK1A) and three miRNAs (miR-103a-3p, miR-10a-5p, and miR-199a-3p) sufficient to discriminate between bladder functional states. Cytokine and immune response pathways, transforming growth factor- $\beta$, nitric oxide signaling, and hypertrophic phosphatidylinositol 3-kinase/AKT signaling pathways were shared among all $\mathrm{BOO}$ phenotypes. Activator protein 1 and NF- $\kappa \mathrm{B}$ were dominant transcription factors, and tumor necrosis factor (TNF)- $\alpha$ was the top upstream regulator. ${ }^{7}$

The primary initiator of inflammation, NF- $\kappa \mathrm{B}$, is a key downstream target of TNF- $\alpha$ that is activated by phosphorylation of I $\kappa \mathrm{B}$ by the I $\kappa \mathrm{B}$ kinase (IKK) complex. ${ }^{18} \mathrm{NF}-\kappa \mathrm{B}$ regulates a vast array of genes, important for immunity, proliferation, inflammation, and tumor development. NF- $\kappa \mathrm{B}$ signaling is closely intertwined with miRNAs, and it induces the expression of several miRNAs, such as $\mathrm{miR}-146 \mathrm{a} / \mathrm{b}$, miR155 , and miR-21, that reciprocally regulate NF- $\kappa \mathrm{B}$ signaling by targeting many of its upstream mediators. ${ }^{19}$ Hypoxia increases the expression of inflammatory cytokines, transforming growth factor- $\beta$ (protein and mRNA), IL-1 $\beta$, IL-6, and TNF- $\alpha$ (mRNA) in human bladder SMCs. ${ }^{20}$ Hypoxia is a hallmark of $\mathrm{BOO},{ }^{21}$ and our transcriptome analysis confirmed these findings, showing the up-regulation of both inflammatory and hypoxic markers in $\mathrm{BOO}$ patients. $^{7}$

Herein, we validated the next-generation sequencing (NGS) and pathway BOO analysis ${ }^{7}$ in cell-based models using primary cultures of human bladder SMCs and urothelial (UE) cells exposed to TNF- $\alpha$. miRNA profiling and transcriptome analysis were performed of TNF- $\alpha$-treated bladder SMCs, and the results were compared with human BOO NGS data to identify the TNF-specific and TNFindependent pathways in the human BOO samples. The expression levels of four abundant TNF-regulated miRNAs were modulated to assess their role in NF- $\kappa \mathrm{B}$ signaling. The important miRNA targets were identified, and their impact on TNF- $\alpha$-driven bladder remodeling is discussed.

\section{Materials and Methods}

\section{Data Depository}

The mRNA-sequencing data set was deposited in the European Nucleotide Archive under European Nucleotide Archive accession number PRJEB24781 (https://www.ebi. ac.uk/ena). The human BOO mRNA- and miRNAsequencing data sets were deposited under European Nucleotide Archive accession numbers PRJEB11369 (for mRNAs) and ERP012260 (for miRNAs). ${ }^{\text {? }}$

\section{Reagents and Antibodies}

Monoclonal antibodies against IKK $\beta$, NF- $\mathrm{B}$, p65, and apoptosis signal-regulating kinase 1 (ASK1) were from Abcam (Cambridge, MA). Monoclonal antibody against smooth muscle (SM) $\alpha$-actin (1A4) (A 2547) was from Sigma-Aldrich (Buchs, Switzerland); and anti- $\beta$-actin (a3854) was purchased from Sigma-Aldrich. Restriction endonucleases, Taq polymerase, and T4 DNA ligase were purchased from New England Biolabs (Ipswich, MA). Chemicals were from Sigma. Recombinant human TNF- $\alpha$ was from Prepotec (London, UK). Cell proliferation enzymelinked immunosorbent assay [5-bromo-2'-deoxyuridine (BrdU)] was from Roche (Rotkreuz, Switzerland). Horseradish peroxidase-conjugated secondary antibodies were from GE Healthcare (Glattbrugg, Switzerland). Papain, 1,4dithioerythritol, bovine serum albumin, collagenase type 1A, and dyspase II were from Sigma-Aldrich. Pre-miR precursors Cy3 dye-labeled negative control (AM17120) and miR-199a5p (ID: PM10893) were purchased from Ambion (Thermo Fisher Scientific, Reinach, Switzerland). Silencer select predesigned siRNAs for mitogen-activated protein kinase kinase kinase (MAP3K5; ID: s8676), inhibitor of nuclear factor $\kappa$ B kinase subunit $\beta$ (IKBKB; ID: s7263), nuclear factor $\kappa$ B subunit 1 (NFKB1; ID: s9504), and phosphatidylinositol-4,5bisphosphate 3-kinase catalytic subunit delta (PIK3CD; ID: s224263) were ordered from Thermo Fisher Scientific. Realtime quantitative PCR (qPCR) gene expression assays were from Thermo Fisher Scientific.

\section{Cell Culture and TNF- $\alpha$ Treatment}

HEK293 cells were maintained in Dulbecco's modified Eagle's medium containing $2 \mathrm{mmol} / \mathrm{L}$ glutamine (Gibco, Thermo Fisher Scientific, Reinach, Switzerland), $100 \mathrm{U}$ of penicillin $/ \mathrm{mL}, 100 \mu \mathrm{g}$ of streptomycin $/ \mathrm{mL}$, and $10 \%$ fetal calf serum (Gibco, Life Technologies). HEK293 cells were transfected using jetPrime (VWR, Dietikon, Switzerland). For primary cultures, samples from the dome were obtained from patients undergoing radical cystectomy for bladder 
cancer, who had no lower urinary tract symptoms aside from hematuria, following informed consent (Canton Bern's Ethics Committee Permit KEK 146/05). The samples localized well away from the tumor. Primary cultures of human bladder UE cells were established and maintained in CnT-18 medium (CELLnTEC, Bern, Switzerland), as described previously. ${ }^{15}$ UE cells were plated on CELL + coated petri dishes (Sarstedt, Switzerland). Primary SMC cultures were established following papaincollagenase protocol. ${ }^{16}$ Cell passages between 1 and 4 were used. All cell cultures were incubated at $37^{\circ} \mathrm{C}$ and $5 \%$ $\mathrm{CO}_{2}$. Before TNF- $\alpha$ treatment, HEK293 cells and SMCs were serum starved for 24 hours using Dulbecco's modified Eagle's medium with $1 \%$ fetal calf serum to induce quiescence and then incubated with recombinant $10 \mathrm{ng} / \mathrm{mL}$ TNF$\alpha$ for the indicated time. UE cells grown in serum-free medium were activated without starving.

\section{Western Blot Analysis}

Cells were washed with $1 \times$ phosphate-buffered saline, scraped using a cell scraper, and lysed in $100 \mathrm{mmol} / \mathrm{L}$ Tris, $20 \mathrm{mmol} / \mathrm{L}$ EGTA, and 5\% SDS, $\mathrm{pH}$ 6.8. Lysates were passed through pipette to shear DNA, boiled, and separated by SDS-PAGE. Proteins were transferred onto a polyvinylidene fluoride membrane, followed by Western blot analysis using chemiluminescence imaging system Fusion FX7-826. The same blots were probed with antibody of choice, followed by control antibody anti-SM $\alpha$-actin in SMCs and anti- $\beta$-actin in HEK293 cells. Images were quantified using ImageJ version 1.51 (NIH, Bethesda, MD; http://imagej.nih.gov/ij), and the optical density of test bands related to control in the same sample.

\section{Cell Proliferation Assays}

Cell proliferation was assessed using BrdU cell proliferation assay (Roche). Primary human bladder SMCs and UE cells were seeded in a 96-well plate and incubated overnight at $37^{\circ} \mathrm{C}$ in a humidified atmosphere containing $5 \% \mathrm{CO}_{2}$ in Dulbecco's modified Eagle's medium with $10 \%$ fetal calf serum in CELL + culture dishes. Cells were treated 24 to 72 hours with $10 \mathrm{ng} / \mathrm{mL}$ TNF- $\alpha$, and $10 \mathrm{mmol} / \mathrm{L}$ BrdU labeling solution was added for 24 hours. BrdU incorporation was detected following manufacturer's protocol and assessed in an enzymelinked immunosorbent assay reader at $370 \mathrm{~nm}$. Data were expressed related to readings of unstimulated cell controls.

\section{Lentivirus Production, Transduction of HEK293, and Primary Human Bladder SMCs}

Plasmids containing hsa-miR-146b-5p (HmiR-AN0197-AM03B), hsa-miR-221-5p (HmiR0369-MR03), hsa-miR-145-5p (HmiR0085-MR03), hsa-miR-21-5p (HmiR-AN0316AM03-B), anti-miR-146b-5p (HmiR0164-MR02), and anti-miR-21-5p (HmiR0284-MR02) were purchased from
GeneCopoeia (Rockville, MD). miR-199a and AM199a-5p were as described previously. ${ }^{16}$ Lentiviruses expressing shRNA and scrambled shRNA control, tagged with mCherry, were purchased from OmicsLink shRNA Expression Clones (GeneCopoeia). pHAGE NFkB-TA-LUC-UBC-GFP-W plasmid $^{22}$ was purchased from Addgene (Cambridge, MA). HEK293T cells (System Biosciences, Palo Alto, CA) were plated at $60 \%$ confluency on $10-\mathrm{cm}$ culture dishes and transfected with $6 \mathrm{mg}$ of each of the lentiviral vectors, 3.25 $\mathrm{mg}$ of packaging pPAX2, and $2 \mathrm{mg}$ of pMD2.G plasmids using jetPrime reagent, following the manufacturer's instructions. The supernatant was collected 24,48 , and 72 hours after transfection and mixed with PEG-it virus concentration solution (System Biosciences) overnight at $4^{\circ} \mathrm{C}$. Viruses were precipitated at $1500 \times g$ at $4^{\circ} \mathrm{C}$ the next day and resuspended in phosphate-buffered saline. Subconfluent cultures of primary human bladder SMCs and HEK293 cells were transduced with recombinant lentiviral particles using $5 \times 10^{6}$ transducing infectious units per $1 \times 10^{6}$ cells in the presence of $8 \mathrm{mg} / \mathrm{mL}$ of polybrene (Sigma-Aldrich). Transduced cells were propagated and used for assays.

\section{NF- $\kappa$ B Activation Luciferase Reporter Assays}

Primary human bladder SMCs and HEK293 cells were cotransduced with the pHAGE NFkB-TA-LUC-UBC-GFP$\mathrm{W} N \mathrm{NF}-\kappa \mathrm{B}$ reporter virus and recombinant lentiviruses expressing scrambled miRNA, or different miRNAs and anti-miRNAs. Cells were serum starved and treated for 24 hours with $10 \mathrm{ng} / \mathrm{mL}$ TNF- $\alpha$. Luciferase activity was detected using luciferase assay (Promega, Madison, WI). HEK293 cells stably expressing NF- $\kappa \mathrm{B}-$ luc reporter were transfected with $10 \mathrm{nmol} / \mathrm{L}$ siRNA or scrambled miRNA control, and luciferase activity was measured 24 hours after transfection following stimulation with $10 \mathrm{nmol} / \mathrm{L}$ TNF- $\alpha$ for 5 hours.

\section{Firefly Luciferase Constructs and Luciferase Reporter Assays}

$3^{\prime}$ Untranslated region (UTR) sequences of human IKBKB and MAP3K5 mRNAs containing putative miR-199a-5p binding sites were PCR amplified from SMC cDNA and cloned into the pmirGLO vector (Promega). For IKBKB, $3^{\prime}$ UTR fragment forward was 5'-ATAAAGCTAGCCCTGACATGGGGCAGCCCATAGCAG-3'; and reverse, 5'-ATAAACTCGAGCCAAAATTGTGCTTTATTAAATGC- $3^{\prime}$. For MAP3 $K 5 \quad 3^{\prime} \quad$ UTR fragment forward was 5'-ATAAAGCTAGCCTGTTGCTCAATCTAATCT-3'; and reverse, $5^{\prime}$ ATAAAGTCGACTATGTTGAGAGTCTCTTTAATTTT-3' Putative miR-199a-5p binding sites of PIK3CD and NFKBI were cloned as linkers into pmirGLO using the following oligos: for PIK3CD 119 target binding site forward, 5'-CTAGCGATATCCTGCCTTTTGTTACACTGGTG-3'; and reverse, 5'-TCGACACCAGTGTAACAAAAGGCAGGATATCG-3'; for NFKB1 202 target binding site forward, 5'-CTAGCGATATCTATCTAGCAATCACAACACTGGCG-3'; and 
reverse, 5'-TCGACGCCAGTGTTGTGATTGCTAGATAGATATCG-3'. Both binding site linkers were annealed and cloned in pmirGLO digested with NheI and SalI. Previously constructed pmirGLO-tgt vector containing the miR199a-5p target sequence 5'-GAACAGGTAGTCTGAACACTGGG-3' was used as a positive control. ${ }^{17}$ Luciferase activity was detected with Dual-Luciferase Reporter Assay kit (Promega), and the activity normalized to Renilla luciferase expressed from the pmirGLO vector.

\section{High-Throughput miRNA NanoString Profiling}

Total RNA (300 ng) was analyzed using the nCounter Human miRNA Expression Assay Kit H_miRNA_V3 (NanoString, Seattle, WA), according to manufacturer's instruction. Each sample was scanned for 555 fields of view using the nCounter Digital Analyzer. nCounter data imaging quality control metrics revealed no significant discrepancy between the fields of view attempted and the fields of view counted. The binding density for the samples ranged between 0.08 and 0.21 within the recommended range. Positive and negative control subtraction and normalization of raw data were performed using NanoStringNorm and NanoStringDiff R packages (available in CRAN), and expression profiling was performed using EdgeR from the R statistical environment version 3.3.2.

\section{Illumina mRNA Sequencing}

mRNA sequencing was performed on the Illumina HiSeq 2000 (Illumina, San Diego, CA) using RNA isolated from bladder SMCs treated with $10 \mathrm{ng} / \mathrm{mL}$ recombinant TNF- $\alpha$ for 24 or 48 hours, as described earlier. ${ }^{7,16}$ High-quality RNA was used for library preparation with the TruSeq RNA Sample Prep Kit v2 (Illumina). Briefly, total RNA samples (1000 ng) were poly(A) enriched and reverse transcribed, and the cDNA samples were fragmented, end repaired, and polyadenylated before ligation of TruSeq adapters containing the index for multiplexing. Fragments containing TruSeq adapters on both ends were selectively enriched with PCR. The TruSeq PE Cluster Kit v3-cBot-HS or TruSeq SR Cluster Kit v3-cBot-HS (Illumina) was used for cluster generation using $10 \mathrm{pmol} / \mathrm{L}$ of pooled normalized libraries on the cBOT. Sequencing was performed on the Illumina HiSeq 2000 single-end 100 bp using the TruSeq SBS Kit v3-HS (Illumina). Read mapping to human reference genome hg19 was done using Tophat 2.0.9. Counting the number of reads/gene was done using HTSeq v.0.5.4p3. The bioconductor package edgeR (Bioconductor version: Release 3.2) was used to identify genes that were differentially expressed comparing the number of mapped reads for each gene in control versus TNF stimulation.

miRNA Target Prediction and miRNA-mRNA Expression Pairing

The predicted target mRNAs of the regulated miRNAs were obtained using TargetScan version 7.0, TarBase, and the experimentally observed relationships are from TarBase. As previously described, the miRNA Target Filter tool in Ingenuity Pathway Analysis (IPA; Qiagen, Redwood City, CA) was used to associate miRNAs from the miRNA sequencing data sets with experimentally observed and highly predicted mRNA targets. ${ }^{7}$ This allows us to prioritize targets using experimental results and information provided by Ingenuity Knowledge Base. The miRNA-mRNA expression pairings function (IPA) was applied to determine the direction of miRNA and mRNA expression changes. The inversely correlated miRNAs and their targets were selected.

\section{Prediction of the Biological Function of Canonical Pathways}

Previously, an $\mathrm{R}$ program tool was developed, which quantifies the involvement of each IPA canonical pathway in Biological Function Classification Database of IPA known as ingenuity canonical pathway. ${ }^{7}$ The number of pathways in mRNA-sequencing data set with a specific biological function is determined and shown as a radar graph.

\section{Z-Score Calculation for Activity of Canonical Pathways}

The pathway activity (z score) was calculated to determine whether the activity of canonical pathways is increased or decreased on the basis of differentially expressed genes in the data sets. On the basis of the calculated z-score, color was then assigned to pathway bar charts to indicate the predicted pathway activity in the data set. The overall activation/inhibition states of canonical pathways are predicted on the basis of a $\mathrm{z}$-score algorithm. The significant values for the canonical pathways were calculated by Fisher's exact test right tailed.

\section{Hierarchical Clustering and Heat Maps}

Hierarchical clustering and the associated heat maps for miRNA and mRNA sequencing data were generated with the function heatmap2 in the R package gplots or GENE-E $\mathrm{R}$ package, as described previously. ${ }^{7}$ On the basis of Pearson correlation method, pairwise correlation matrix between items was computed and converted to a distance matrix; finally, clustering was computed on the resulting distance matrix. Average linkage method used average to calculate the distance matrix. To draw simple Venn diagrams without size adjustments, Venny and jvenn were used. ${ }^{23}$

\section{Gene Clouds (Tag Clouds and Word Clouds)}

To condense and visualize large amounts of gene enrichment data from a pathway analysis data set and find biological patterns, a cloud was generated with Wordle.net and Word 
cloud $\mathrm{R}$ package. The font size of a gene (tag) is determined by its incidence in the pathway analysis data set. ${ }^{7}$

\section{Quantitative RT-PCR}

Total RNA was isolated using the miRVana miRNA isolation kit (Ambion). The reverse transcription reactions were performed using the High Capacity cDNA Reverse Transcription Kit (Applied Biosystems, Foster City, CA) with random hexamer primers. TaqMan assays were from Applied Biosystems. Quantitative RT-PCR was performed in triplicate using 7900HT Fast Real-Time PCR System (Applied Biosystems). The Ct values obtained after the qPCR were normalized to the $18 \mathrm{~S}$ rRNA when performing TaqMan qPCR gene expression assays.

\section{Statistical Analysis}

The significance for the canonical pathways was calculated by Fisher's exact test right tailed. A one-way analysis of variance was used to compare the relative quantities of the mRNAs in quantitative RT-PCR. The Tukey correction was used to correct $P$ values. $P<0.05$ was considered as statistically significant (GraphPad Prism version 6.0; GraphPad Software, La Jolla, CA).

\section{Treated SMCs}

A

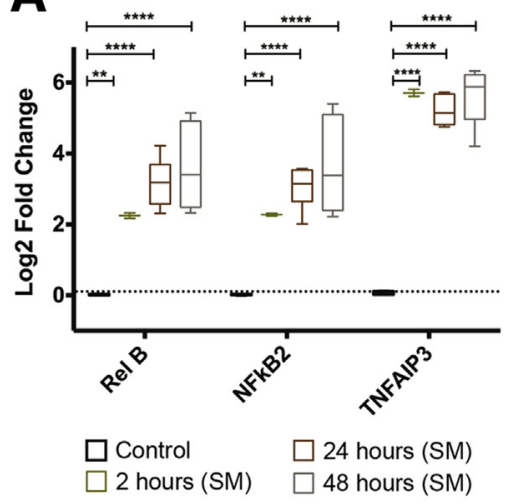

B

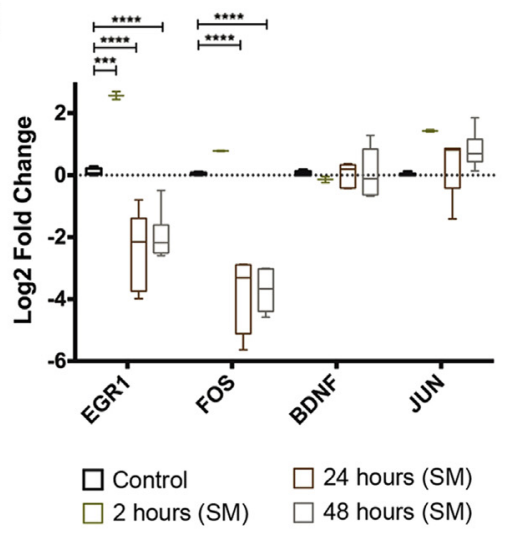

Treated UE Cells

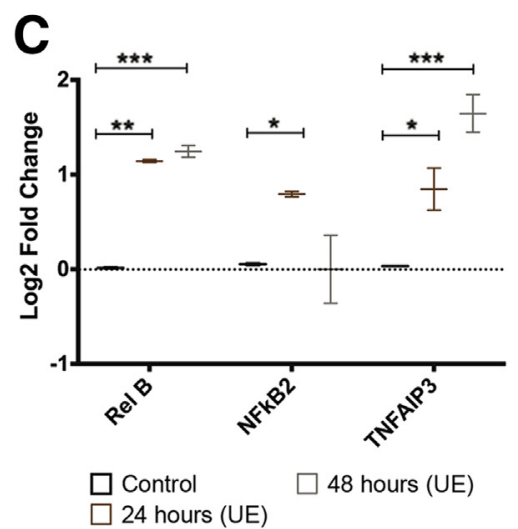

D

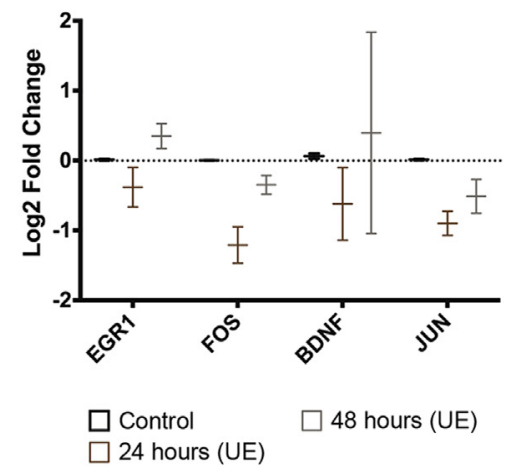

BOO Patients

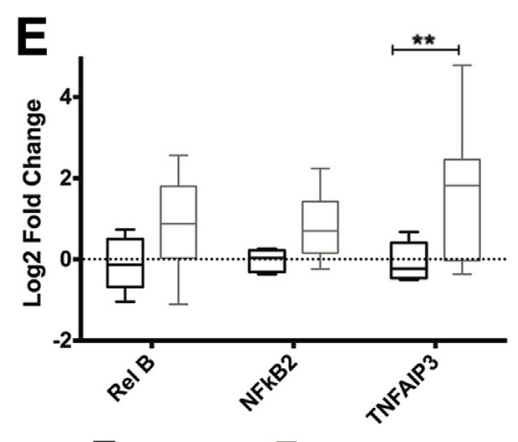

F

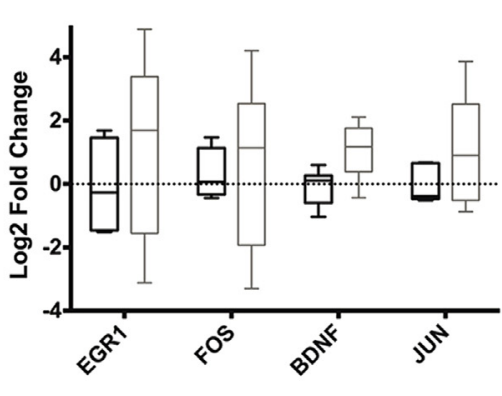

$\square$ Control

BOO Patients

Figure 1 Tumor necrosis factor (TNF)- $\alpha-$ regulated mRNAs in the bladder smooth muscle cells (SMCs) and urothelial (UE) cells and in bladder outlet obstruction (B00). Bladder SMCS and UE cells were treated with $10 \mathrm{ng} / \mathrm{mL}$ TNF- $\alpha$, as described in Materials and Methods. Gene expression levels were analyzed by quantitative RT-PCR and shown as log2 fold changes compared with untreated controls. Dotted lines represent no difference with control. RelB, NFKB2, and TNFAIP3 in SMCS (A); EGR1, FOS, BDNF, and JUN in SMCS (B); RelB, NFKB2, and TNFAIP3 in UE cells (C); EGR1, FOS, BDNF, and JUN in UE cells (D); RelB, NFKB2, and TNFAIP3 in B00 patients compared with control (E); and EGR1, FOS, BDNF, and JUN in B00 patients (F). Data in $\mathbf{E}$ and $\mathbf{F}$ were extracted from next-generation sequencing. ${ }^{7}$ Data are expressed as means \pm SD of three experiments, each performed in triplicate $(\mathbf{A}-\mathbf{F}) . n=18$ (E, B00 patients); $n=6$ (E, control). ${ }^{*} P<0.05$, ${ }^{* * P}<0.01,{ }^{* * * P}<0.001$, and ${ }^{* * * * P}<0.0001$. 


\section{Results}

Human Bladder Smooth Muscle and Urothelial Cells Respond to TNF- $\alpha$ Treatment by Differential Regulation of miRNAs

Cultures of normal human detrusor SMCs and UE cells were established, as described previously. ${ }^{11,16}$ The cells were treated with $10 \mathrm{ng} / \mathrm{mL}$ of TNF- $\alpha$ for 2,24 , and 48 hours (SMCs) or 24 and 48 hours (UE cells); RNA was isolated, and levels of selected genes were examined by quantitative RT-PCR. TNF-responsive RelB, NFKB2, and TNFAIP3 were chosen to control for the effect of TNF on gene expression. The dynamics of the early $(E G R l)$ and late (JUN and FOS) genes, ${ }^{24}$ as well as the expression levels of $B D N F$, reported to be induced by TNF- $\alpha,{ }^{24}$ were also checked. In SMCs (Figure 1A) and UE cells (Figure 1C), $\operatorname{RelB}, N F K B 2$, and TNFAIP3 were robustly up-regulated at all tested time points, similar to their expression in human BOO (Figure 1E). EGRI, FOS, and JUN showed an increase in SMCs after 2 hours of TNF- $\alpha$ stimulation, with the subsequent decrease or return to the basal level (Figure 1B). $B D N F$, implicated in bladder overactivity ${ }^{25}$ and slightly upregulated in human $\mathrm{BOO}$ (Figure 1F), did not show regulation after TNF treatment of SMCs (Figure 1B) or UE cells (Figure 1D).

Having ensured that both SMC and UE cell cultures were responsive to TNF- $\alpha$ treatment, 800 miRNAs were profiled using NanoString miRNA expression arrays. Abundant miRNAs, robustly expressed in all samples after normalization, were selected (Figure 2A and Supplemental Tables S1-S3). Of all regulated miRNAs, 17 were shared between UE cells and SMCs at all tested time points (Figure 2A). Of these, miR-146a-5p, miR-222-3p, miR-30a5p, miR-7-5p, miR-30e-5p, miR-31-5p, miR-1260a, and miR-7f-5p were significantly $(P<0.05)$ up-regulated by TNF- $\alpha$ treatment; miR-1180, miR-199a-3p, miR-199a-5p, and miR-199b-3p were down-regulated. Five miRNAs (miR-361-5p, miR-582-5p, miR-424-5p, miR-27a-3p, and miR-503-5p) were increased in UE cells and decreased in SMCs (Figure 2B), pointing to the possible differences in activation responses in these bladder layers. Three miRNAs (miR-141-3p, miR-135b-5p, and miR-220b-3p) were significantly (up to 10 -fold read number differences) more abundant in UE cells (Figure 2C), whereas 14 were enriched in SMCs (Figure 2D); most SMC miRNAs showed a decrease after TNF- $\alpha$ treatment. To evaluate the clinical relevance of the observed changes, the miRNA profiles of
A
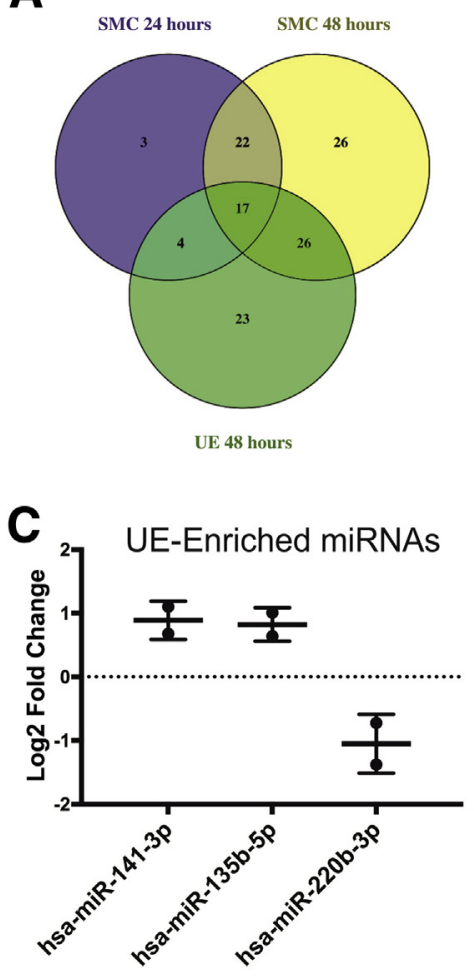

B

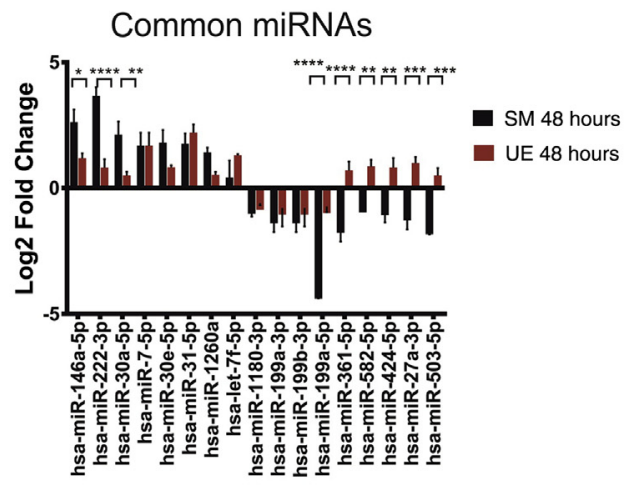

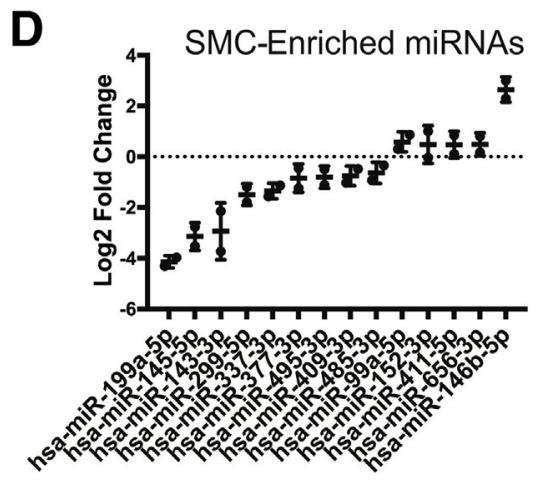

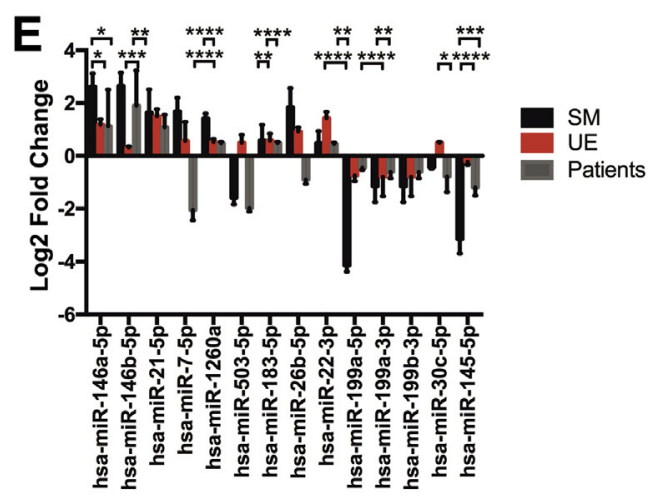

Figure 2 miRNA profiling of tumor necrosis factor (TNF)- $\alpha$-treated bladder smooth muscle cells (SMCs) and urothelial (UE) cells. A: Venn diagram of shared miRNAs, significantly regulated in SMCs after 24 and 48 hours of TNF- $\alpha$ and in UE cells after 48 hours of TNF- $\alpha(10 \mathrm{ng} / \mathrm{mL})$. B: Expression of 17 common miRNAs in SMCs (average of four samples, 48 hours) and UE cells (average of three samples, 48 hours); all differences were significant compared with respective untreated controls. C: UE-enriched (at least 10 -fold read increase compared with SMC, controls) regulated miRNAs. Dotted line represents no difference with control. D: SMC-enriched (at least 10-fold read increase compared with UE, controls) regulated miRNAs. Dotted line represents no difference with control. E: Regulation of shared miRNAs compared with bladder outlet obstruction. Data are expressed as means \pm SD. $n=18(\mathrm{E})$. ${ }^{\star} P<0.05$, $* * P<0.01,{ }^{* *} P<0.001$, and ${ }^{* * * * P}<0.0001$. 
TNF-treated bladder SMCs and UE cells were compared with miRNAs, regulated in human $\mathrm{BOO}$ identified previously. ${ }^{7}$ Indeed, 14 regulated miRNAs were also affected by BOO in human bladders, and only miR-7-5p showed discordant regulation, being induced by TNF- $\alpha$ in the cell culture but down-regulated in human BOO (Figure 2E). miRNAs miR-146a-5p, miR-146b-5p, miR-21-5p, miR183-5p, and miR-22-3p, significantly up-regulated in both the cells and the human BOO (Figure 2E), were previously reported to be induced by TNF- $\alpha .^{19,26,27}$ Taken together, these results indicate the similarities of gene expression changes during $\mathrm{BOO}$ and in TNF- $\alpha$-treated bladder cells.

\section{TNF- $\alpha$ Induces Bladder Cell Proliferation and Decreases the Expression of Contractile Proteins}

TNF- $\alpha$ is a pleiotropic cytokine capable of inducing both cell proliferation and apoptosis, ${ }^{28,29}$ and it has been shown to regulate vascular and airway SMC proliferation. ${ }^{30}$ The growth responses in TNF-treated UE cells and SMCs were examined, which showed that it causes sustained increase in UE cell proliferation already after 24 hours (Figure 3A). SMCs responded with a delay, however, after 48 and 72 hours of $10 \mathrm{ng} / \mathrm{mL}$ TNF- $\alpha$ treatment; BrdU incorporation in SMCs was over twofold stronger than in the untreated cells (Figure 3B).

SMC proliferation is often accompanied by a switch from the contractile to a synthetic phenotype, and the levels of SM markers and differentiation-relevant transcription cofactors were determined in SMCs 24 and 48 hours after application of $10 \mathrm{ng} / \mathrm{mL}$ TNF- $\alpha$ (Figure 3C). With the exception of smoothelin (SMTN), all examined genes encoding SM contractile proteins (SM-specific myosin heavy chains, SM-actin, caldesmon, SM calponin, and transgelin) were significantly down-regulated, together with down-regulation of myocardin and myocardin-dependent genes (MYL9, ACTG2, CDKN1A, and TGFB1I1). In parallel, expression levels of $N R P 2$, SRF-associated factor $E L K$ 1 , and $K L F 4$ were induced (Figure 3C). Although there was a down-regulation of $M Y C$ and $C T G F$, the expression levels of FOSL1, FOSL2, and JUN, as well of other proliferationspecific markers (CCND1, ETS1, and SERPINE1), were significantly increased after 24 and 48 hours of TNF- $\alpha$ treatment (Figure 3D).

\section{Expression Profiles of Stimulated SMCs Reflect Activation of TNF- $\alpha$-Mediated Signaling Pathways}

Although BOO affects all bladder layers, most functionrelevant changes (hypertrophy, fibrosis, and loss of contractility) occur in the detrusor muscle. To obtain a comprehensive image of the functional consequences of TNF- $\alpha$ stimulation, NGS was used to determine gene expression profiles of SMCs, treated for 24 and 48 hours. This approach also allows studying the expression of all predicted miRNA target genes. Differentially expressed mRNAs were determined (absolute fold change $\geq 2$ times, adjusted $P<0.05$ ) (Supplemental Tables S4 and S5). Hierarchical clustering of mRNAs revealed high expression profile similarity between the TNF-treated cells, which were significantly different from the untreated controls (Figure 4A). The overall number of differentially expressed
A
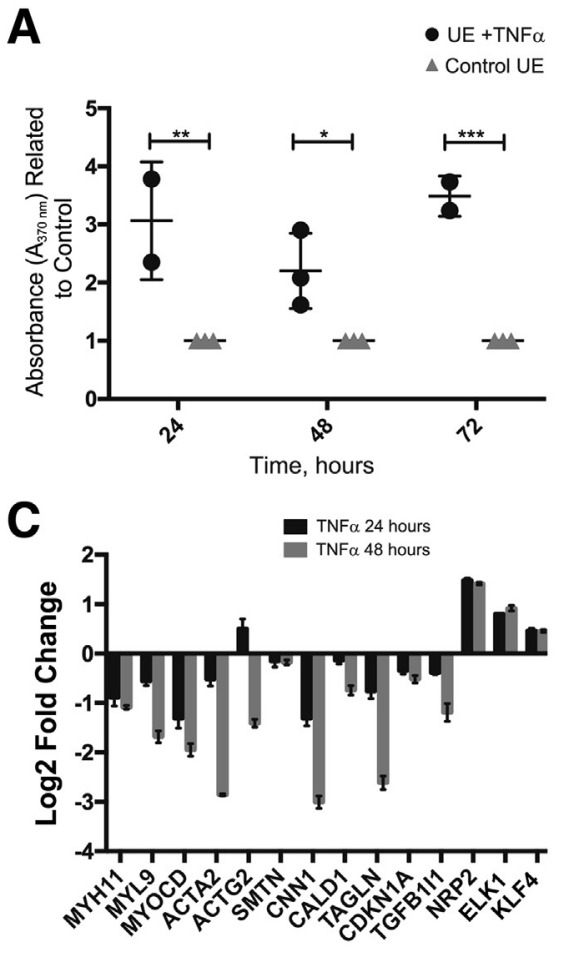

B

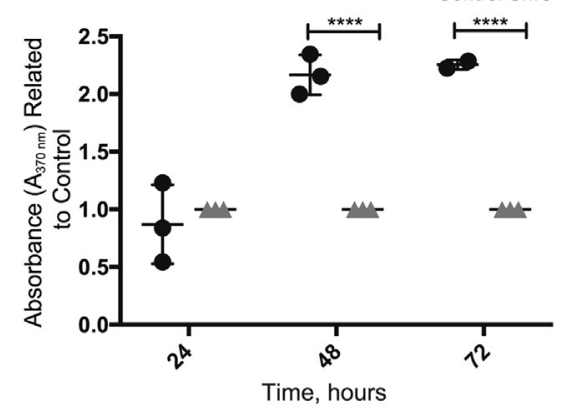

D

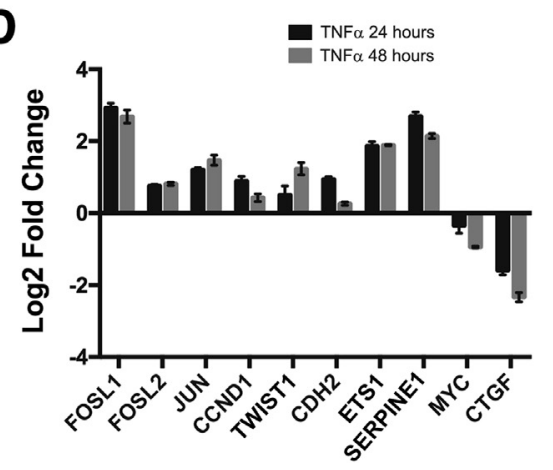

Figure 3 Tumor necrosis factor (TNF)- $\alpha$ induces contractile to synthetic switch in the bladder smooth muscle cells (SMCs). Bladder cells were treated with TNF- $\alpha$, and proliferation was measured by 5 -bromo-2'-deoxyuridine incorporation at indicated time points. A: Proliferation of primary urothelial (UE) cells. B: Proliferation of primary SMCs. Gene expression levels in SMCs treated for 48 hours with TNF- $\alpha$ were analyzed by quantitative RT-PCR and shown as log2 fold changes compared with untreated controls. C: Contractile proteins, SM markers, and relevant transcription factors. D: Markers of proliferation and fibrosis. Data are expressed as means \pm SD of three experiments, each performed in sixfold repetitions (A-D). ${ }^{*} P<0.05,{ }^{*} P P<0.01$, $* * * P<0.001$, and $* * * * P<0.0001$. 
A

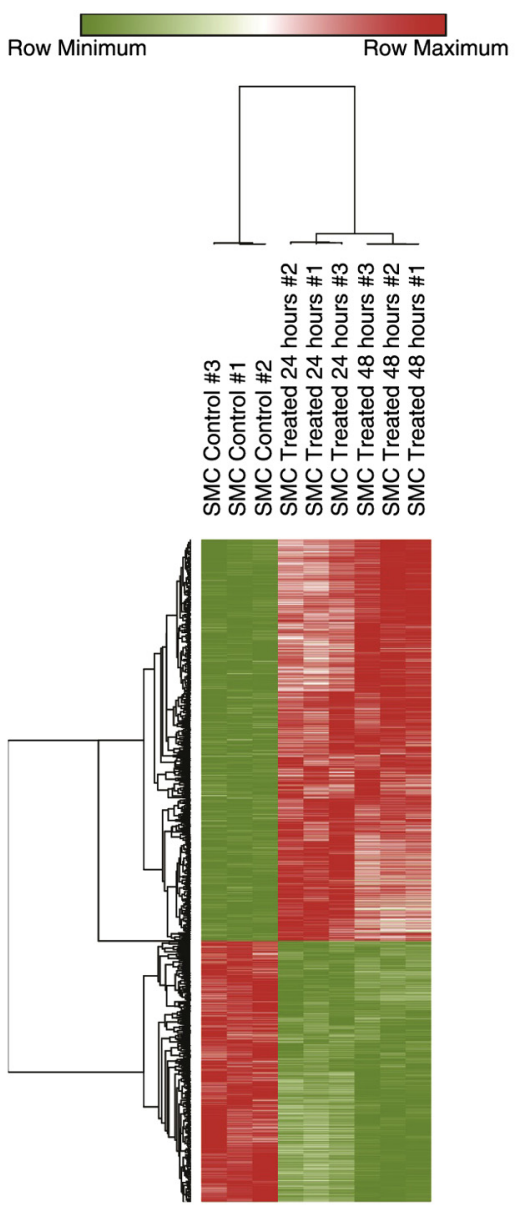

B

\section{SMC 24 hours SMC 48 hours}

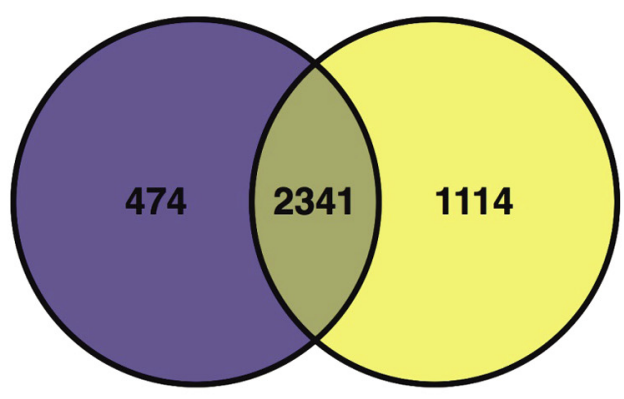

mRNAs

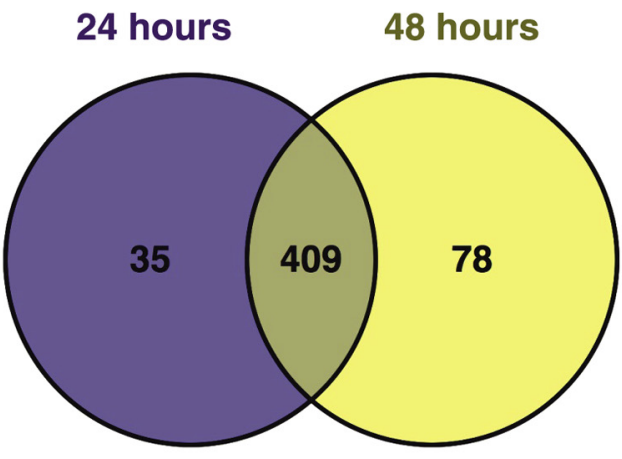

Pathways

\section{Positive Z Score $\square$ Z Score $=0$ Negative Z Score $\square$ No Activity Patterm Avalable $\square$ - Ratio}

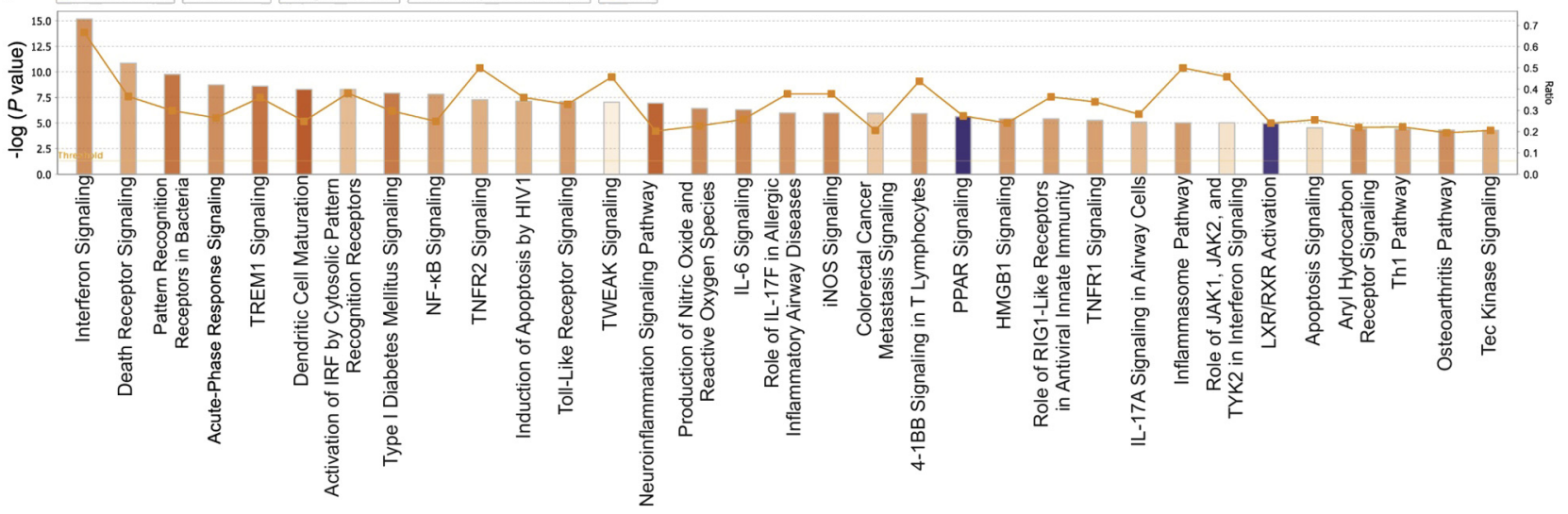

Figure 4 Next-generation sequencing and pathway analysis of smooth muscle cells (SMCs) confirms activation of tumor necrosis factor (TNF)-induced signaling pathways. A: Hierarchical clustering and heat map of log2 fold change of significantly regulated mRNA ( $y$ axis) in SMC controls and SMCs treated with $10 \mathrm{ng} / \mathrm{mL}$ TNF- $\alpha$ for 24 and 48 hours ( $x$ axis). B: Venn diagram showing overlapping significantly regulated mRNAs and pathways in 24- and 48-hour time-point samples. C: Top 33 significant pathways with z scores built using intersecting mRNAs. $n=3$ (A, all groups). inOS, inducible nitric oxide synthase; Max, maximum; Min, minimum; PPAR, peroxisome proliferator-activated receptor; Th1, type 1 helper T cell; TNFR, TNF receptor.

mRNAs increased from 2815 transcripts at 24 hours, to 3455 regulated mRNAs at 48 hours, with 2341 genes shared at both time points (Figure 4B). Similarly, when using each data set individually for pathway analysis, and comparing the outcome, overwhelming 409 pathways were shared between the time points (Figure 4B).

Having determined that the intersecting regulated mRNAs were representative of TNF- $\alpha$-stimulated 
A
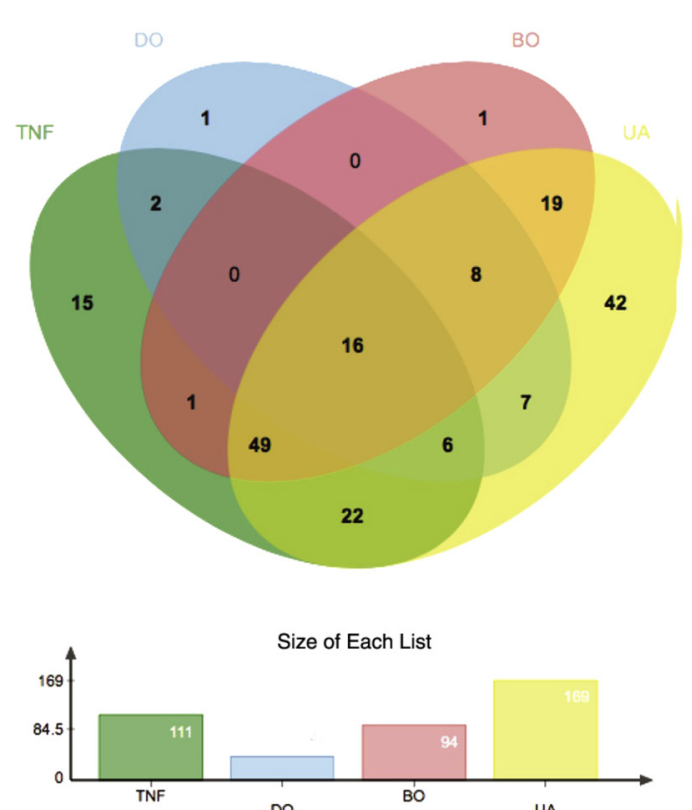

DO

\section{C}

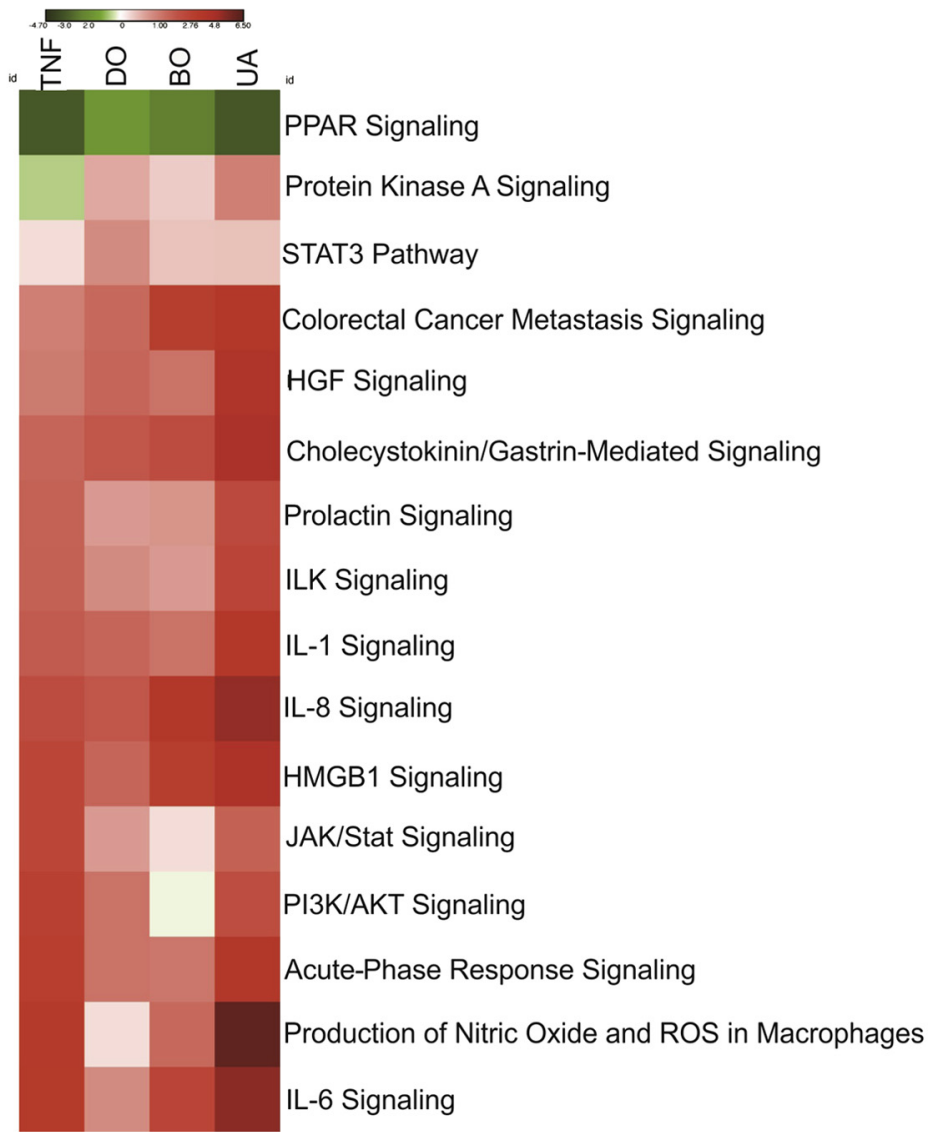

B

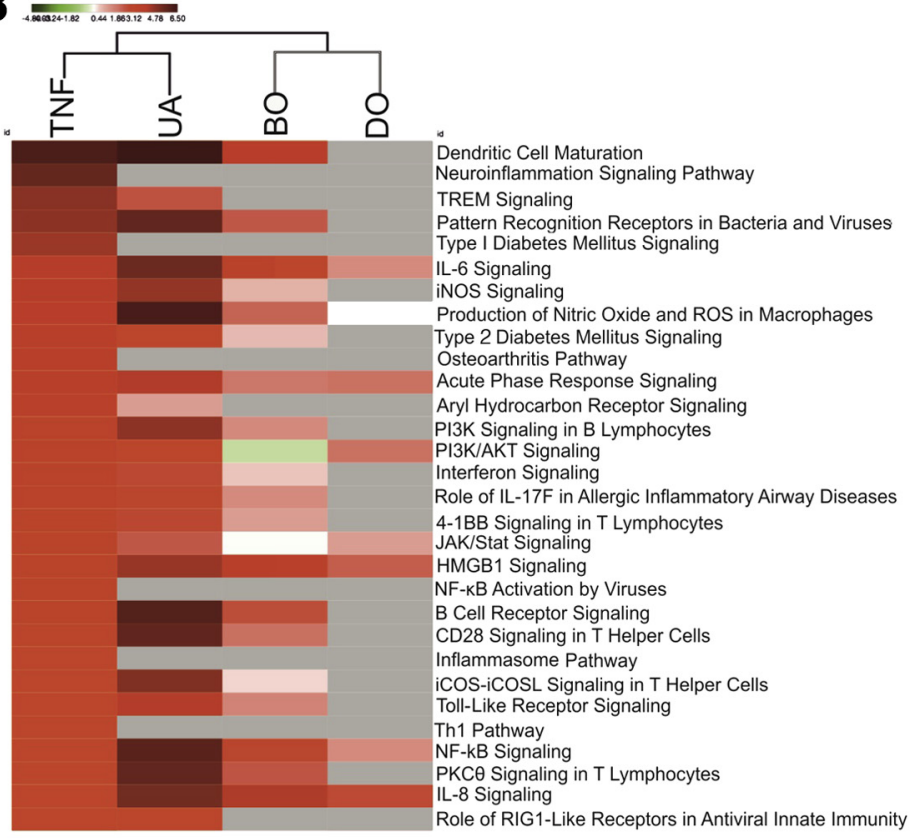

Figure 5 Comparative analysis of tumor necrosis factor (TNF)-induced signaling in smooth muscle cells (SMCs) and bladder outlet obstruction (B00) phenotypes. A: Venn diagram showing an overlap between pathway lists of TNF-treated SMCs (designated TNF, 111 pathways) and B00 phenotypes determined previously, ${ }^{7}$ designated detrusor overactivity (D0; 40 pathways), B00 without DO (B0; 94 pathways), and detrusor underactivity (UA; 169 pathways). B: Heat map and clustering on the basis of $z$ scores of TNF-regulated pathways in SMCs (TNF) pathways. On the basis of $z$ scores of the TNF-regulated pathways in B00 phenotypes, UA clusters with SMCs (designated TNF), and BO clusters with D0. Gray boxes represent the missing pathways. Top 30 pathways are shown; for complete list, refer to Supplemental Figure S1. C: Heat map and clustering on the basis of $z$ scores of 16 common pathways. $Z$ scores of the pathways common in all B00 phenotypes (B0, D0, and $\mathrm{UA}$ ) resemble the scores in TNF-treated SMCs (TNF), with the exception of protein kinase A signaling and STAT3 pathway. iNOS, inducible nitric oxide synthase; PI3K, phosphatidylinositol 3kinase; PKC, protein kinase C; PPAR, peroxisome proliferator-activated receptor; ROS, reactive oxygen species; Th1, type 1 helper T cell.
SMCs (Supplemental Table S6), the gene enrichment analysis was refined, including $\mathrm{z}$ scores to reflect the activation status, and $P<0.05$ (Supplemental Table S7). The top 33 canonical pathways based on regulated mRNAs are shown in Figure 4C. With the exception of two pathways [peroxisome proliferator-activated receptor signaling and liver X nuclear receptor/retinoid X receptor (LXR/RXR) activation], all other pathways show activation. 
A

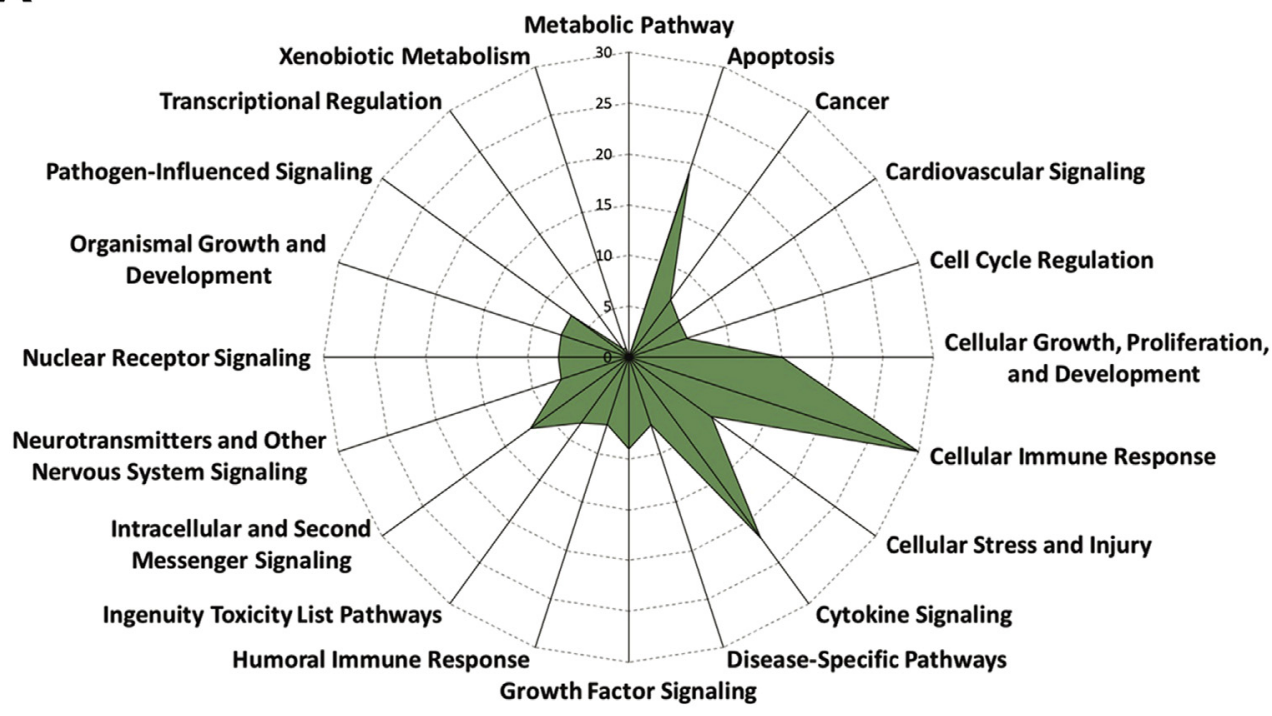

B

- No TNF (DO, BO, UA)

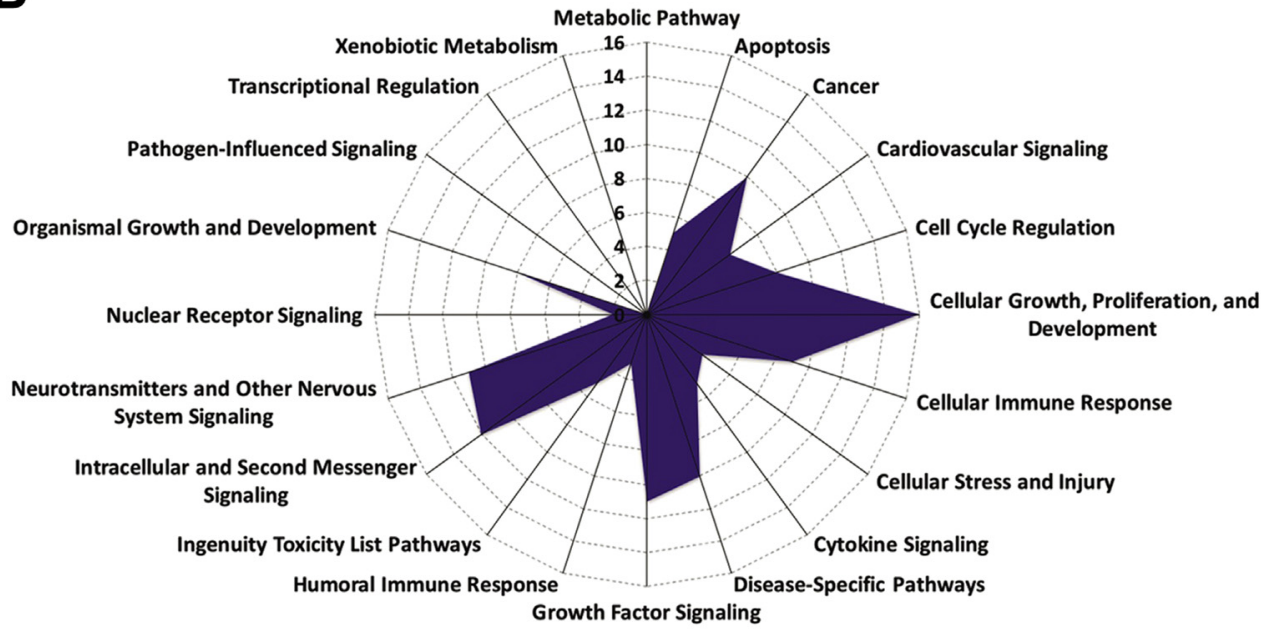

C

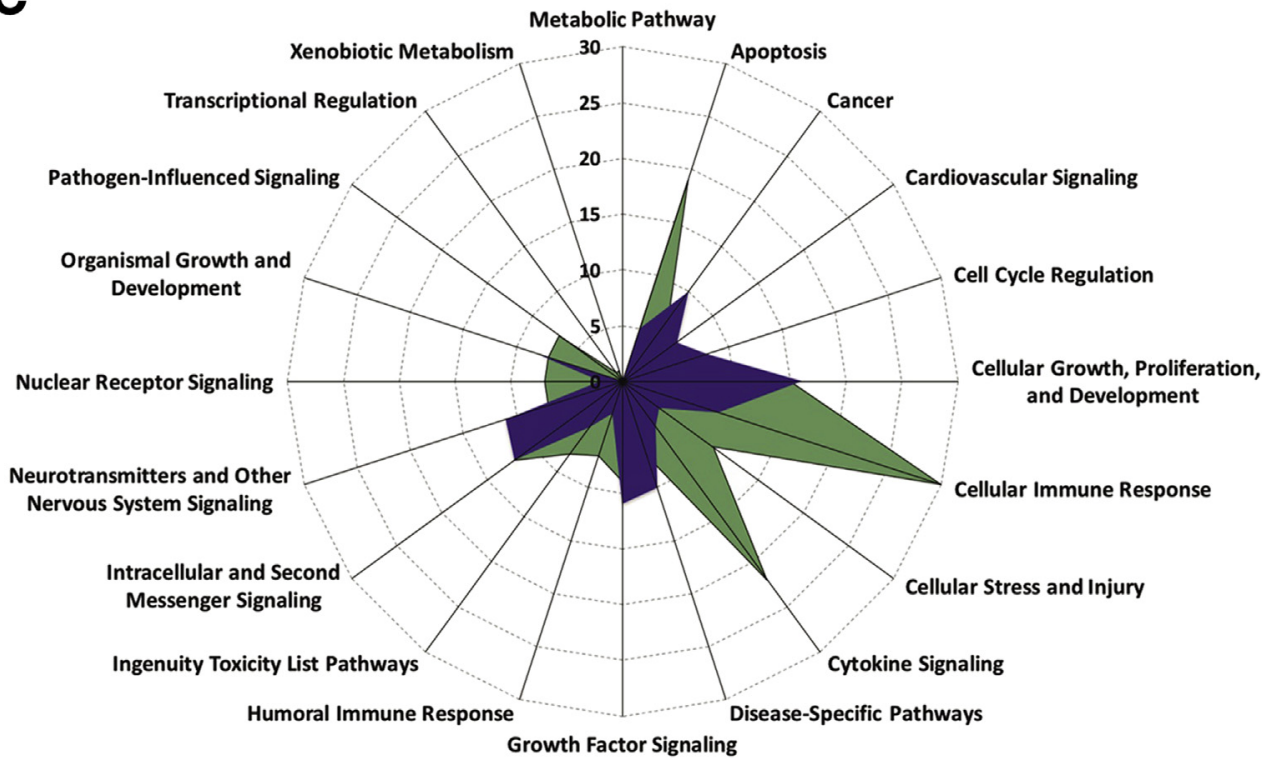


Comparative Pathway Analysis of TNF- $\alpha$-Stimulated SMCs and B00 Patient Groups with Different Functional Phenotypes

The pathway analysis results from the in vitro assays were compared with previously published patient data. ${ }^{7}$ The nomenclature of the patient groups and the selection criteria is restated for clarity. BOO patients were recruited and examined as described. ${ }^{7}$ On the basis of the urodynamic examination, the patients were divided into the following (phenotype) groups: group 1, designated control or $\mathrm{C}$ (normal bladder function); group 2, designated DO (BOO with DO); group 3, designated $\mathrm{BO}$ (BOO without $\mathrm{DO}$ ); and group 4, designated UA (BOO with detrusor acontractility). The pathway analysis was previously performed using differentially expressed mRNAs determined by NGS from each phenotypic BOO group (absolute fold change $\geq 1.5$ times, $P<0.05$, adjusted $P<0.15$ ). One hundred and eleven TNFdriven pathways were compared in the bladder SMCs, with 40, 94, and 169 pathways regulated in DO, BO, and UA, respectively. Venn analysis showed a varying number of intersecting elements, with 16 pathways shared between TNF-stimulated SMCs and all BOO groups (Figure 5A).

To assess the impact of TNF-driven signaling in each BOO phenotype, regulated pathways were hierarchically clustered (Figure 5B and Supplemental Figure S1). On the basis of the activation score and the number of identical/ similarly regulated pathways, UA was clustered together with TNF- $\alpha-$ treated SMCs, followed by BO. DO had the least similarity in pathway activation. Previously, 22 pathways shared between all BOO phenotypes were identified. ${ }^{7}$ At least 14 of them were found to be dependent on TNF- $\alpha$ signaling (Figure 5C). Interestingly, protein kinase A signaling was inhibited in TNF-stimulated SMCs (negative z score) but activated in human BOO. Similarly, the STAT3 pathway was high in BOO groups and almost not regulated in SMCs, on the basis of $\mathrm{z}$ score (Figure 5C). Both of these pathways are relevant for hypertrophy, which prompted us to define and compare the biological functions of TNFregulated and TNF-independent pathways in BOO.

A recently developed bioinformatic tool was used to attribute biological functions to the pathways, regulated in different data sets, which allows for their quantitative and functional comparison. ${ }^{7}$ The main functions of TNFregulated signaling pathways were cellular immune response, followed by cytokine signaling and apoptosis (Figure 6A). The biological functions of the pathways, built using the whole BOO biopsy mRNA data $\operatorname{set}^{7}$ (Supplemental Figure S2A), show high similarity with TNF- $\alpha$ signaling pathways, established in bladder SMCs in vitro (Supplemental Figure S2B). On the other hand, after excluding the pathways, overlapping in treated SMC and BOO data sets, the TNF-independent biological functions could be determined (Figure 6B). These included cellular growth, proliferation, and development, growth factor signaling, second messenger signaling, and neurotransmitter signaling. The overlap of TNF-regulated and TNFindependent processes is shown in Figure 6C.

\section{Compensatory Up-Regulation of miR-199a-5p Reduces NF- $\kappa B$ Signaling}

Abundant miRNAs, most likely to have biological activity, ${ }^{31}$ were selected to test whether modulation of their levels by overexpression or inactivation with anti-miRNAs affected $\mathrm{NF}-\kappa \mathrm{B}$ signaling downstream of TNF- $\alpha$. NF- $\kappa \mathrm{B}-$ driven luciferase gene was used as a reporter, and assays were performed in HEK293 cells and primary SMCs transduced with lentiviruses overexpressing miR-199a-5p, miR-145-5p, miR21-5p, miR-146b-5p, or their respective anti-miRNAs. Two SM-specific significantly TNF-inhibited miRNAs (miR199a-5p and miR-145-5p) and two TNF-induced ubiquitously expressed miRNAs (miR-21-5p and miR-146b-6p) were selected, because they showed similar regulation patterns in UE and SMC in vitro assays and in BOO. Of all of the miRNAs tested, only miR-199a-5p produced significant effects. Its overexpression in both HEK293 cells (Figure 7A) and SMCs (Figure 7B) caused decrease of NF- $\kappa \mathrm{B}$ activation, whereas its inhibition with anti-miRNA did not affect the signaling, because miR-199a-5p had already been strongly inhibited by TNF- $\alpha$ (Figure 2, D and E).

Bioinformatics prediction was performed for all miR$199 \mathrm{a}-5 \mathrm{p}$ expressed and appropriately regulated targets, and 157 up-regulated target mRNAs were used for pathway analysis (Figure 7C). In agreement with its previously described role as a negative regulator of SMC proliferation, ${ }^{16}$ the up-regulated targets of TNF repressed miR-199a-5p, negatively regulated $\mathrm{G}_{1} / \mathrm{S}$ cell cycle checkpoint, and significantly contributed to the activated TNF- $\alpha$-driven pathways, including NF- $\kappa$ B signaling (Figure $7 \mathrm{C}$ ).

Top signaling molecules were identified in SMCs treated with TNF- $\alpha$ using Word Cloud and Biological Function Classification Database (Supplemental Figure S3A). The word cloud of frequent elements from 111 significant pathways of differentially expressed mRNAs contains all of the major effectors of NF- $\kappa \mathrm{B}$ signaling and other TNF- $\alpha$-driven processes (Supplemental Figure S3A). A similar word cloud of miR199a-5p targets involved in signaling pathways is shown in Supplemental Figure S3B; it contains important hubs of TNF$\alpha$-activated processes, such as NFKB1, PIK3CD, and MAP3K5 (ASK1). We hypothesized that down-regulation of these molecules by miR-199a-5p overexpression might

Figure 6 Biological functions assigned to tumor necrosis factor (TNF)-dependent and TNF-independent pathways in bladder outlet obstruction (B00) patients. A: The radar graph for biological functions of significant $(P<0.05)$ TNF smooth muscle cell mRNA data set pathways (green). B: The radar graphs for biological functions of 105 pathways built using mRNA data sets of all B00 phenotypes, which show no overlap with TNF pathways (blue). C: TNF-driven (green, TNF) and TNF-independent (blue, no TNF [detrusor overactivity (D0), B00 without D0 (B0), detrusor underactivity (UA)]) graphs superimposed. 

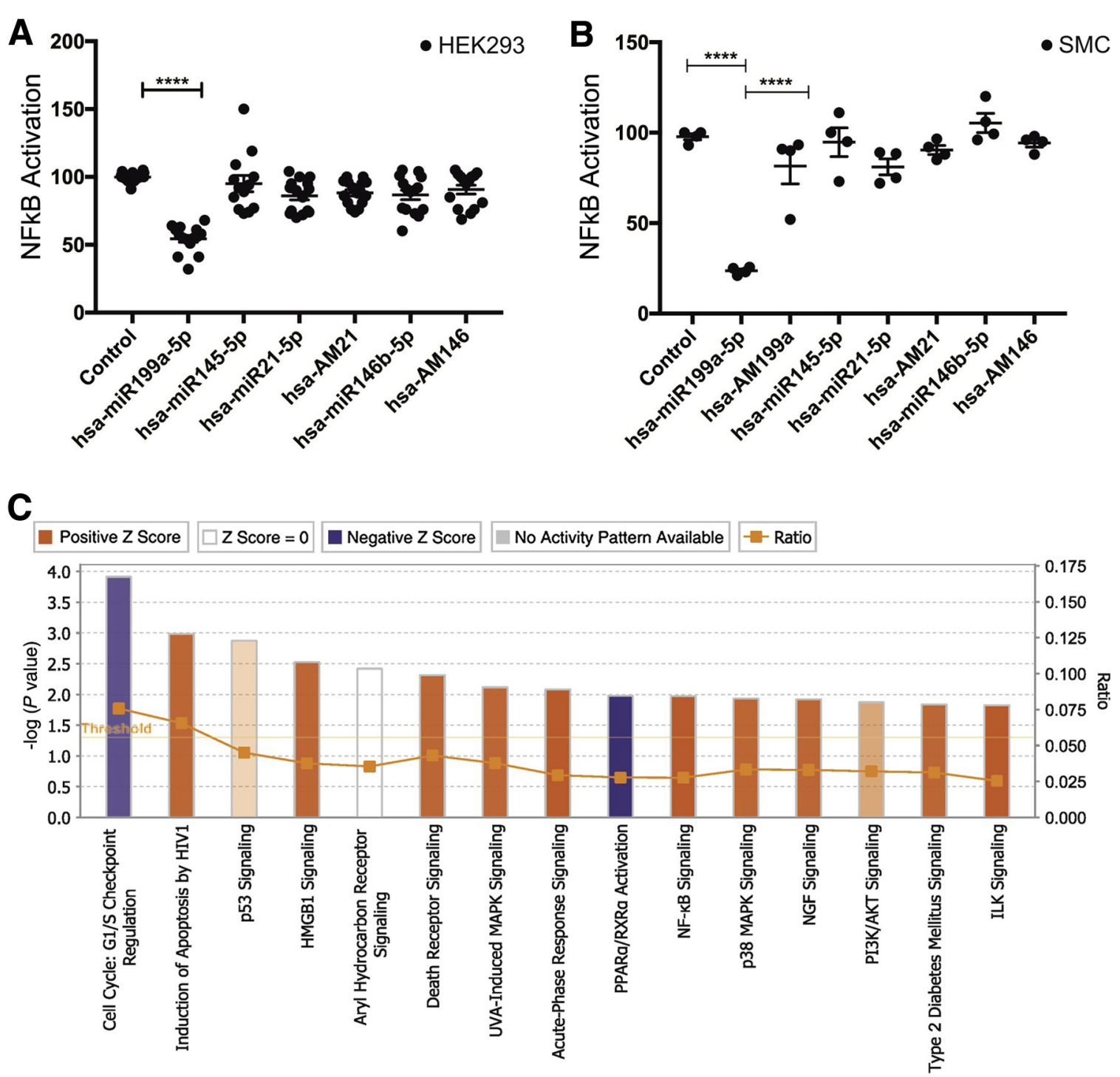

Figure 7 Overexpression of miR-199a-5p reduces NF- $\mathrm{B}$ signaling. Using NF- $\kappa \mathrm{B}-$ driven luciferase gene as a reporter, cells transduced with lentiviruses overexpressing miR-199a-5p, miR-145-5p, miR-21-5p, miR-146b-5p, or their respective anti-miRNAs were stimulated with tumor necrosis factor (TNF)- $\alpha$ and NF-KB activation calculated relative to control. The data are average of HEK239 cells and smooth muscle cells (SMCs) performed in sixfold repetition. A: HEK293 cells were used to overexpress miRNAs or anti-miRNAs. No anti-miR-199a-5p was used because these cells lack the endogenous miR-199a-5p. B: Bladder SMCs overexpressing miRNAs and anti-miRNAs; only miR-199a-5p overexpression significantly reduces NF- $\kappa$ B signaling. C: Pathways built using miR199a-5p targets, up-regulated in TNF- $\alpha$-treated SMCs. Pathways are significant $(P<0.05)$, with a z-score prediction. $n=15$ (HEK239 cells); $n=4$ (SMCs). $* * * P<0.0001$. HMGB, high mobility group box; ILK, integrin linked kinase; MAPK, mitogen-activated protein kinase; NGF, nerve growth factor; PI3K, phosphatidylinositol 3-kinase; PPAR, peroxisome proliferator-activated receptor; RXR, retinoid $\mathrm{X}$ receptor.

interfere with downstream activation of $\mathrm{NF}-\kappa \mathrm{B}$ observed in HEK293 cells and SMCs (Figure 7, A and B).

Striving to explain the lack of effect of the other three miRNAs on NF- $\mathrm{KB}$ signaling, the contribution of their respective mRNA targets to the pathways activated by TNF- $\alpha$ was determined. For each tested miRNA, the total number of appropriately regulated targets was counted in the mRNAsequencing data set, and the number of pathway elements among them was determined. miR-199a-5p had the highest number of appropriately regulated targets and pathway elements, compared with the other three miRNAs (Figure 8A). Appropriately regulated mRNA targets were predicted for miR-21-5p and miR-146b-5p (down-regulated in the mRNA sequencing data set) and for miR-145-5p (up-regulated in the mRNA data set) and mapped against all of the TNF signaling pathway elements. Indeed, miR-199a-5p targets included more abundant and more influential signaling hubs (Supplemental Figure S4A) than miR-145-5p (Supplemental Figure S4B), miR-21-5p (Supplemental Figure S4C), or miR146b-5p (Supplemental Figure S4D) targets. The occurrence of the top five pathway elements, targeted by the tested miRNAs in 111 significant $(P<0.05)$ TNF- $\alpha$ signaling pathways, is shown as a bubble graph in Figure 8B. Upregulated targets of miR-199a-5p have the highest occurrence in TNF-regulated pathways, pointing at their regulatory significance. 

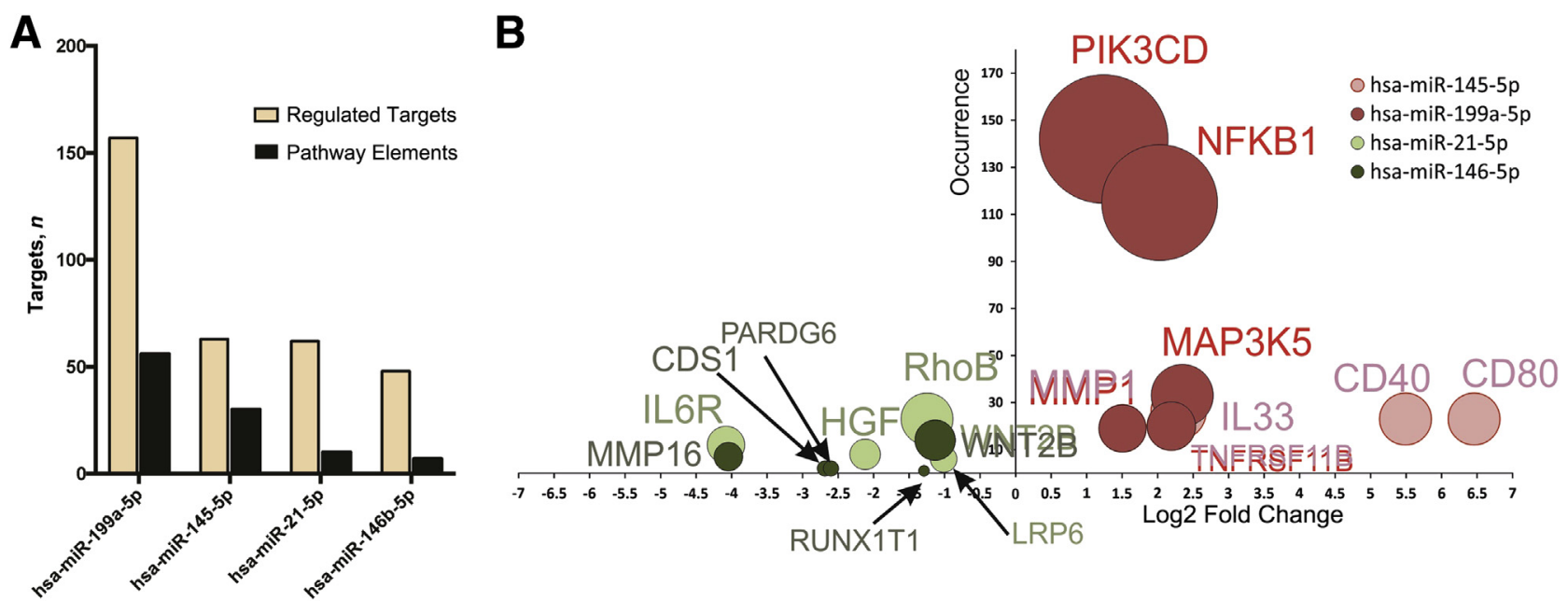

Figure 8 miRNA targets among tumor necrosis factor (TNF) signaling pathway elements. A: Graph showing all appropriately regulated targets and the number of pathway elements among them for each tested miRNA. B: Bubble graph shows the occurrence of the top five pathway elements, targeted by the upregulated miRNAs miR-21-5p and miR-146b-5p and the down-regulated miR-199a-5p and miR-145-5p in 111 significant $(P<0.05)$ TNF- $\alpha$ signaling pathways. The pathway elements, predicted to be targeted by a particular miRNA and appropriately regulated in the data set, are shown as a data point. Diameter of each data point reflects frequency of occurrence of the element ( $y$ axis). $x$ Axis represents log2 fold change expression of miRNA target genes relative to scrambled control. Up-regulated mRNA targets are shown in red (dark red for miR-199a-5p and light red for miR-145-5p). Down-regulated targets are shown in green (dark green for miR-146b-5p and light green for miR-21-5p).

Experimental Validation of IKBKB and MAP3K5 as miR199a-5p Targets, Critically Involved in the Activation of NF-KB Signaling

In addition to the mRNAs shown in Supplemental Figure S3A, IKK $\beta$ encoded by the $I K B K B$ gene is a protein crucial for NF- $\mathrm{KB}$ signaling. ${ }^{32,33} I K B K B$ is not regulated by TNF- $\alpha$ and, thus, was absent from our data sets; however, it was previously shown to be targeted by miR-199a-5p in cancer cells. ${ }^{34,35}$ Therefore, it was included with the other three primary mRNA candidates (NFKB1, PIK3CD, and MAP3K5) in the analysis of NF$\kappa \mathrm{B}-$ relevant miR-199a-5p targets. The putative miR-199a-5p binding site sequences in the $3^{\prime}$ UTRs of all candidates are shown in Supplemental Figure S5. The mRNA levels of these genes were assessed in SMCs, overexpressing miR-199a-5p by quantitative RT-PCR, and the results compared with the previous NGS data in TEU-2 urothelial cells, transfected with pre-miR199a. ${ }^{17}$ Only $I K B K B$ and MAP3K5 showed significantly $(P<0.05)$ decreased mRNA levels in both cell types, in the presence of miR-199a-5p, whereas there was no effect on NFKB1 or PIK3CD mRNA levels (Figure 9A). Next, it was tested whether compensatory up-regulation of miR-199a-5p reduces the levels of these mRNAs during TNF- $\alpha$ stimulation of SMCs. As expected, MAP3K5, NFKB1, and PIK3CD were increased in SMCs treated with $10 \mathrm{ng} / \mathrm{mL}$ TNF- $\alpha$ for 48 hours, and further reduction of endogenous miR-199a-5p levels in these cells with anti-miR-199a-5p induced higher up-regulation of $M A P 3 K 5$ and NFKB1. The compensatory up-regulation of miR199a-5p by transduction with miR-199a-expressing lentivirus significantly attenuated their TNF-induced up-regulation (Figure 9B). In line with NGS data, the basal levels of IKBKB were not affected by TNF stimulation, but were significantly reduced in the presence of miR-199a-5p (Figure 9B).

miRNA interaction with the putative binding sites expressed as the luciferase $3^{\prime}$ UTR in the pmirGLO vector was validated, as described previously, ${ }^{11,16}$ and compared with the pmirGLO with an miR-199a-5p target as positive control. A significant down-regulation of luciferase activity was observed in cells expressing binding sites ( $3^{\prime}$ UTRs) of $I K B K B$ and MAP3K5 (Figure 9C) in the presence of miR199a-5p, but there was no such inhibition for NFKB1 and PIK3CD (Supplemental Figure S6A). Similarly, the protein levels of IKK $\beta$ and MAP3K5 (ASK1) were significantly reduced in SMCs overexpressing miR-199a-5p compared with the scrambled control (Figure 9D). On the other hand, the protein levels of NFKB1 were not affected by miR199a-5p (Supplemental Figure S6B). PIK3CD could not be assessed because of the lack of reliable antibodies.

Having established that miR-199a-5p targets and significantly down-regulates $I K B K B$ and $M A P 3 K 5$, it was determined whether reducing the expression levels of each or both of these proteins affected NF- $\mathrm{B}$ signaling. HEK293 cells, stably expressing an NF- $\kappa \mathrm{B}-$ regulated luciferase reporter construct, were transfected with siRNAs specifically knocking down IKBKB and/or MAP3K5, and significant $(P<0.0001)$ reduction of TNF- $\alpha$-induced NF- $\kappa$ B activation was observed (Figure 9E). Both siRNAs decreased the protein levels of IKK $\beta$ and ASK1, as determined by Western blotting (Figure 9F). These data confirm the important role of miR$199 \mathrm{a}-5 \mathrm{p}$ targets $I K B K B$ and $M A P 3 K 5$ in NF- $\kappa \mathrm{B}$ signaling, and help explain the mechanism of miRNA-induced attenuation of the TNF- $\alpha-$ mediated response. 
A mRNA in SMCs

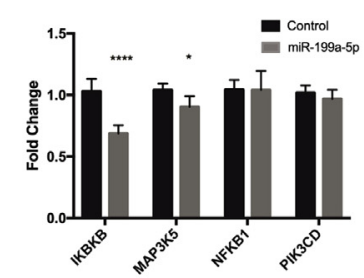

mRNA in TEU2

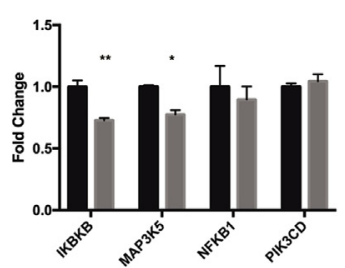

B Regulation by TNF $\alpha 48$ hours

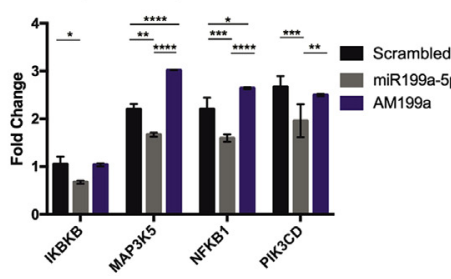

C

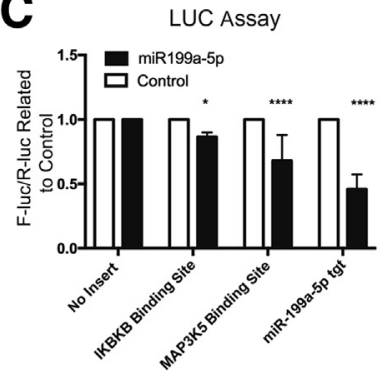

D

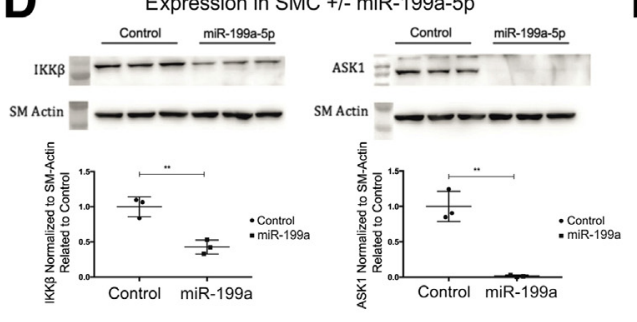

$\mathbf{E}$

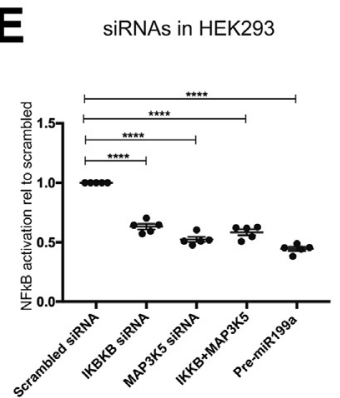

$\mathbf{F}$

Expression in HEK293 +/- siRNA

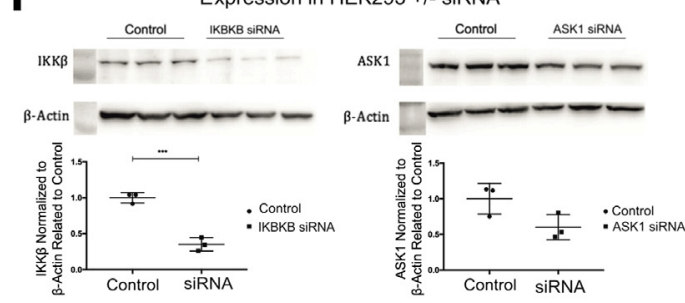

Figure 9 Experimental validation of IKBKB and mitogen-activated protein kinase kinase kinase (MAP3K5) as targets of miR-199a-5p in smooth muscle cells (SMCs), influencing NF- $K B$ signaling. A: mRNA levels of IKBKB, MAP3K5, NFKB1, and PIK3CD were determined by quantitative RT-PCR (RT-qPCR) in SMCs overexpressing miR-199a-5p and compared with control (scrambled miRNA) (top graph, mRNA in SMCS). In TEU-2 next-generation sequencing data set, $^{25}$ the reads of pre-miR-199a transfected cells were compared with controls. All results are expressed as fold change related to values in scrambled controls; means of triplicates are shown. B: SMCs expressing miR-199a, anti-miR-199a-5p, or scrambled miRNA control were treated with $10 \mathrm{ng} / \mathrm{mL}$ tumor necrosis factor (TNF)- $\alpha$ at 48 hours, and mRNA levels of IKBKB, MAP3K5, NFKB1, and PIK3CD were determined by RT-qPCR. Data are expressed as fold change relative to untreated controls for each gene and cell type. Means of triplicates are shown. C: $3^{\prime}$ Untranslated region sequences of $I K B K B$ (IKBKB binding site) and MAP3K5 (MAP3K5 binding site), as well as the perfectly complementary binding site for miR-199a-5p (miR-199a-5p tgt) were cloned into pmirGLO luciferase reporter vector and luciferase activity assays, performed as described in Materials and Methods in HEK293 cells overexpressing miR-199a or scrambled miRNA control. Renilla luciferase activity expressed from the same vector was used for normalization, and the data are expressed relative to the negative control. D: Bladder SMCs were stably transduced with lentiviruses expressing control scrambled miRNA and miR-199a-5p, and protein levels of IKB kinase $\beta$ (IKK $\beta$ ) and apoptosis signal-regulating kinase 1 (ASK1) were analyzed by Western blotting. SM $\alpha$-actin was used as loading control. Quantification analysis was performed as described in Materials and Methods, and values were normalized to SM actin for each sample shown. The graph shows an average of three experiments. Statistically significant differences between scrambled control and miR-199a-5p-expressing SMCs are indicated. E: HEK293 cells stably transduced with a lentivirus expressing NF- $K B$-regulated luciferase were transiently transfected with siRNAs against IKBKB, MAP3K5, or a mixture of both. Cy-3-labeled scrambled miRNA was used as a negative control and pre-miR-199a as a positive control. Twenty-four hours after transfection, cells were stimulated with TNF- $\alpha$ and expression of luciferase was measured as readout for NF- $\mathrm{KB}$ activation. All graphs show an average of five experiments performed in sixfold repetition. Statistically significant differences compared with the vector without insert are indicated. F: HEK293 cells were transfected with scrambled miR-Cy3 or siRNAs against IKBKB and MAP3K5, and protein levels of IKK $\beta$ and ASK1 analyzed by Western blotting with $\beta$-actin as loading control. The graph shows values normalized to $\beta$-actin in three experiments. Statistically significant differences between control and siRNA transfected cells are indicated. Data are expressed as means \pm SD $(\mathbf{A}, \mathbf{E}$, and $\mathbf{F})$ or means \pm SEM (D). ${ }^{*} P<0.05,{ }^{*} P<0.01,{ }^{* * *} P<0.001$, and ${ }^{* * * * P}<0.0001$ versus control. IKBKB, inhibitor of nuclear factor $\kappa \mathrm{B}$ kinase subunit $\beta$.

\section{Discussion}

The goals of this study were to validate the NGS and pathway analysis of the bladder biopsy specimens from the humans with different phenotypes of urodynamically defined BOO-induced bladder dysfunction ${ }^{7}$ and to determine the role of TNF- $\alpha$ in the regulation of bladder remodeling. Transcriptome data analysis revealed TNF- $\alpha$ as the top upstream regulator in all analyzed data sets (BOO and individual groups) and NF- $\mathrm{BB}$ together with activator protein 1 (JUN-FOS) as the main transcription factors. The primary cultures of bladder SMCs and UE cells were treated with TNF- $\alpha$ to define the impact of this cytokine on the overall mRNA and miRNA expression profiles and compare the in vitro findings with data obtained in BOO.
Herein, an in-depth analysis of gene expression changes (miRNA by NanoString arrays and mRNA by NGS) occurring in bladder SMCs exposed to TNF- $\alpha$ was performed, and these data were correlated with our previous BOO transcriptome analysis to study its influence on bladder remodeling. ${ }^{7} \mathrm{TNF}-\alpha$ was found to be a strong modulator of miRNA expression in both bladder SMCs and UE cells, causing a profound dysregulation of their synthesis. Seventeen miRNAs were identified, which were changed in both cell types at all tested time points, 12 of them showing concordant regulation in UE cells and SMCs and 14 also regulated in BOO. Many of the changes observed with BOO were modeled in vitro in UE cells and SMCs treated with TNF- $\alpha$. A cellbased approach, although not without its limitations, allowed pairing the regulated miRNAs with their targets and 


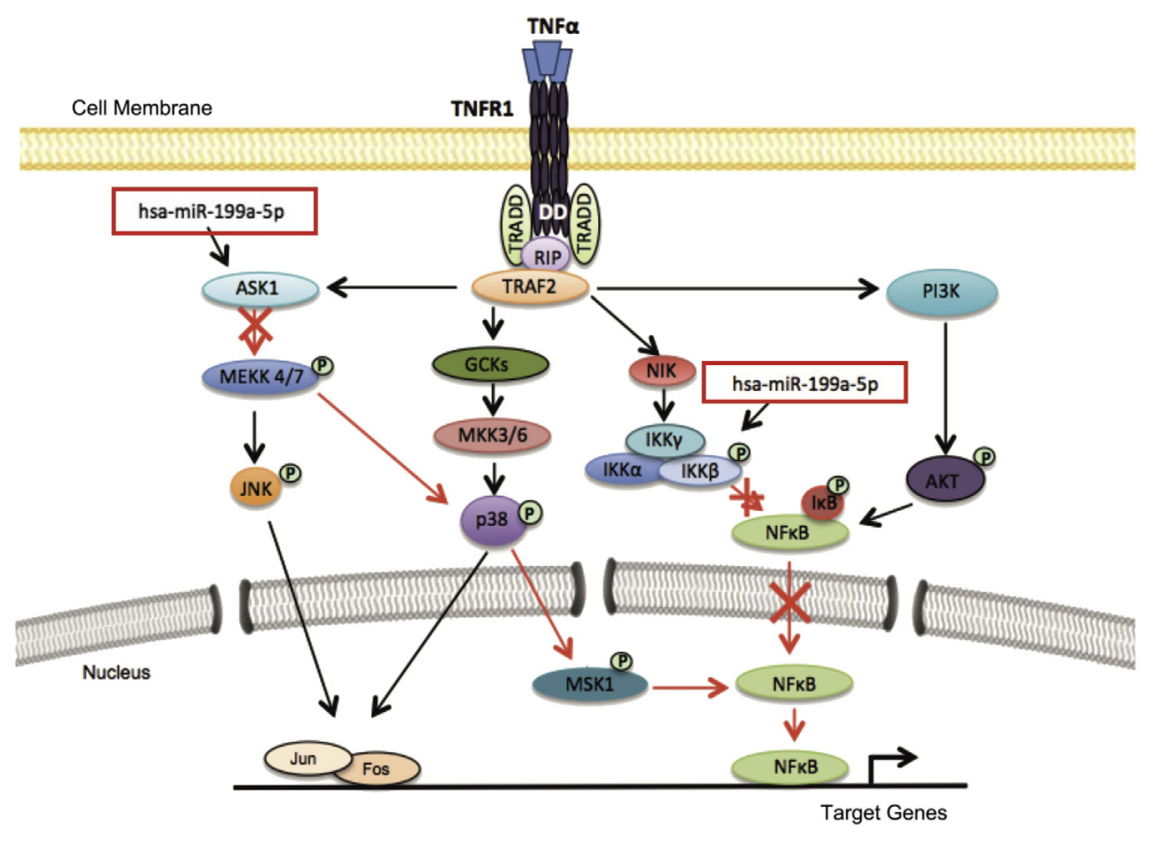

Figure 10 Modulation of tumor necrosis factor (TNF)- $\alpha$ signaling by miR-199a-5p. miR-199a$5 p$ (red boxes) binds to its target mRNAs and down-regulates the expression of apoptosis signal-regulating kinase 1 (ASK1) and I $B$ kinase $\beta$ (IKK $\beta$; red crosses), causing subsequent reduction of $\mathrm{NF}-\mathrm{KB}-$ and JUN-FOS-mediated gene expression. JNK, c-Jun $\mathrm{N}$-terminal kinase; PI3K, phosphatidylinositol 3-kinase; TNFR1, TNF receptor 1. determining the influence of selected miRNAs on cell signaling. miR-199a-5p, previously described as a regulator of SM differentiation and contractility, ${ }^{16}$ is an important inhibitor of NF- $\kappa \mathrm{B}$ signaling via its targets IKK $\beta$ and ASK1 (Figure 10). Other SMC-specific miRNAs were downregulated after TNF- $\alpha$ treatment, in parallel with an increase of SMC proliferation and a significant down-regulation of contractile markers. On the other hand, TNF- $\alpha$ stimulation was accompanied by a significant up-regulation of $N R P 2$, $E L K-1, K L F 4$, and activator protein 1 transcription factor subunits JUN and FOSL1/FOSL2 (Figure 10). Transcription factor ELK-1, whose expression is regulated by NF- $\kappa B$, ${ }^{36}$ together with KLF4 suppress the expression of SM genes. ${ }^{16,37}$ Interestingly, expression levels of neuropilin 2 (NRP2) also increased TNF dependently. NRP2 was recently identified as a prorelaxant stimulus in SMCs, ${ }^{38}$ and its downregulation was observed in $\mathrm{BOO}$ concomitant with an increase in bladder pressure during voiding in hypertrophic bladders. ${ }^{39}$ Although there was a down-regulation of $M Y C$ and $C T G F$, the expression levels of FOSL1, FOSL2, and $J U N$, as well of other proliferation-specific markers (CCND1, ETS1, and SERPINE1), were significantly increased after TNF- $\alpha$ treatment. These findings are in accordance with the down-regulation of SM markers and loss of contractility in human bladders and implicate TNF- $\alpha$ in inducing the switch between contractile and synthetic smooth muscle phenotypes.

Transcriptome analysis of TNF-treated SMCs was performed, and as expected, pathway analysis revealed activation of inflammatory and immune pathways. Our goal was to compare the cell signaling profiles of TNF- $\alpha$-treated SMCs with the pathways regulated in phenotypes of BOO and assess the impact of TNF- $\alpha$ on the signaling events during BOO. A discrepancy was observed between the functional phenotypes regarding TNF- $\alpha$ signaling: DO had the lowest number of TNF-driven pathways, followed by BO. The highest number of TNF-regulated pathways was observed in UA, demonstrating an increasing importance of TNF- $\alpha$ signaling during the progression of bladder dysfunction from hypertrophy to decompensation. Gradual decrease of bladder contractility and increasing fibrosis, leading to acontractile phenotype, are well documented in the animal studies. ${ }^{6}$ Longitudinal studies in humans are less clear, showing both the incidence of clinical progression with symptom worsening, ${ }^{40}$ as well as no significant urodynamic deterioration. ${ }^{41}$ Progressive worsening of LUTS and $\mathrm{BOO}$ secondary to benign prostatic hyperplasia might be the result of multiple causes, including androgen receptor signaling, inflammatory cytokines, and growth factor signals. ${ }^{42}$ There is a considerable variability between patients: a significant minority of patients in the study by Thomas et $\mathrm{al}^{41}$ has experienced a gradual deterioration of contractility. Underactive bladder syndrome is a complex multifactorial condition that shares symptoms with other urologic diagnoses. Nevertheless, at least in some cases, which might include BOO-induced LUTS, it has been hypothesized that chronic untreated or treatment refractory overactive bladder syndrome attributable to bladder outlet obstruction may progress to detrusor hyperreflexia/impaired contractility and, finally, bladder underactivity. ${ }^{43}$ In the original study, the UA group represented obstructed acontractile bladder patients. $^{7}$ The magnitude of gene expression changes was increasing from $\mathrm{DO}$ to $\mathrm{BO}$ and $\mathrm{UA}$ groups, making the disease progression in case of BOO-induced LUTD an attractive possibility. In this study, the comparative approach has allowed delineating the TNF-independent processes during BOO. Having subtracted the 
TNF-activated pathway signature of SMCs from the full list of pathways, activated during BOO, the processes of cell growth, proliferation, and development, growth factor, and second messenger signaling remained, which were not shared between the TNF-treated SMCs and BOO. On the basis of the higher number of proliferative pathways in DO, its weak TNF- $\alpha$ pathway signature, and the observed increase of TNF signaling pathways in BO and UA, it is tempting to speculate that the impact of TNF- $\alpha$ increases as obstruction progresses. Of 22 pathways, 14, shared in all BOO phenotypes, were found to be $\mathrm{TNF}-\alpha$ regulated. Peroxisome proliferator-activated receptor signaling was predicted to be inhibited by TNF- $\alpha$, in line with its antiinflammatory and antiproliferative effects. ${ }^{44}$

Proinflammatory cytokines are released by the SMCs subjected to hypoxia ${ }^{20}$ or mechanical force, ${ }^{45}$ and attract immune cells, particularly macrophages, which are an important source of TNF- $\alpha{ }^{46}$ TNF- $\alpha$ released by macrophages plays a major role in diabetic renal injury ${ }^{47}$ and other fibrotic diseases. ${ }^{48} \mathrm{~A}$ recent study confirmed induction of inflammatory genes and macrophage infiltration in the obstructed bladder and showed that bladder SMCs subjected to hydrostatic pressure released IL-6, monocyte chemoattractant protein 1 (MCP1), and regulated on activation normal $\mathrm{T}$ cell expressed and secreted (RANTES). ${ }^{49}$ Indeed, in human BOO patients, a significant up-regulation of MMD, MPEG1, CSR1R, CCL2, and other markers was observed, indicating monocyte infiltration and macrophage differentiation. ${ }^{7}$

Interestingly, the miRNA profiling in $\mathrm{TNF}-\alpha-$ treated SMCs and UE cells showed up-regulation of many miRNAs previously implicated in the fibrotic and hypertrophy responses, notably miR-21 and miR-146a/b. ${ }^{50}$ These profibrotic miRNAs were also up-regulated in $\mathrm{BOO}$, indicating a cross talk between inflammatory processes and bladder fibrosis. Concomitant with the loss of contractile markers, observed in TNF-treated SMCs, the expression levels of SMspecific miRNAs, including miR-143/145 and miR-199a-5p, were reduced while the same changes were observed in BOO. Not all contractility defects in BOO were TNF modulated: muscle-specific miRNAs miR-1 and miR-133, which were down-regulated in BOO, in line with the hypertrophic and fibrotic response, ${ }^{12}$ were not affected in the in vitro assays presented herein. miRNAs have a multitude of functions in health and disease, and mounting evidence suggests that they play a particularly important role in regulating gene expression in response to physiological and pathologic stress. ${ }^{51}$ Acting on their targets, they often form feedback loops serving to sustain the signaling events, which have induced their up-regulation. For example, miR-21-5p, induced by NF$\kappa \mathrm{B}$, in turn inactivates phosphatase and tensin homolog, an inhibitor of AKT phosphorylation, thus promoting NF- $\kappa \mathrm{B}$ activation. ${ }^{52}$ To test this hypothesis, the impact of four TNFregulated miRNAs was assessed on the downstream NF- $\kappa \mathrm{B}$ signaling. miR-199a-5p and miR-145-5p were downregulated, and miR-21-5p and miR-146b-5p were upregulated, in SMCs, UE cells, and BOO. Their expression levels were modulated, and compensatory up-regulation of miR-199a-5p was seen to significantly decrease NF- $\kappa$ B signaling, whereas the other miRNAs had no effect. To account for these differences, the contribution of the respective mRNA targets of each of the tested miRNAs to the pathways activated by TNF- $\alpha$ was determined. miR-199a-5p had the highest number of appropriately regulated targets and pathway elements, compared with the other three miRNAs, and its bioinformatically predicted targets included the influential signaling hubs, such as NFKB1, PIK3CD, IKBKB, and MAP3K5. The experimental validation of miR199a-5p binding to the $3^{\prime}$ UTRs and/or binding sites of $I K B K B$ and $M A P 3 K 5$ confirmed the bioinformatic prediction. Both IKK $\beta$ and ASK1 proteins were significantly downregulated on miR-199a-5p overexpression, and their specific inhibition by siRNAs reduced $N F-\kappa B$ activation. In contrast, $N F K B 1$ and $P I K 3 C D$ could not be validated as miR199a-5p targets, which contradicts an earlier report for PIK3CD in breast cancer cells, ${ }^{53}$ and may be because of cell type specificity of miRNA-target interaction.

The MAP3K5 gene encodes ASK1, a member of the MAP3K family, which activates downstream MAP kinases, c-Jun N-terminal kinases, and p38 MAP kinases. ${ }^{54}$ Herein, for the first time, we identify MAP3K5 as a direct target of miR-199a-5p and show that its inhibition by miR-199a-5p or by a specific siRNA significantly reduces $N F-\kappa B$ activity. Although c-Jun N-terminal kinase/p38 signaling leading to apoptosis is considered the primary outcome of ASK1 activation, there are several reports pointing to its involvement in $\mathrm{NF}-\kappa \mathrm{B}$ signaling. Indeed, in endothelial cells, the p38-dependent pathways directly regulate the transcriptional activity of NF- $\kappa \mathrm{B}$ factors. ${ }^{55} \mathrm{TNF}-\alpha-$ induced TNF receptor associated factor 2 (TRAF2) influences ASK1, ${ }^{29}$ leading to activation of c-Jun N-terminal kinase, which, on one hand, induces activator protein 1, but can also phosphorylate and activate NF- $\kappa$ B. ${ }^{56}$ Downstream of ASK1, the activation of p38 MAP kinase also results in NF- $\kappa \mathrm{B}-$ driven transcription. ${ }^{57}$ Supporting our observations, a knockdown of ASK1 partially inhibited the induction of IL-6 after stimulation with $\mathrm{TNF}-\alpha,{ }^{58}$ which is an $\mathrm{NF}-\kappa \mathrm{B}-$ dependent process. Both IKK $\beta$, identified earlier in cancer, ${ }^{34,35}$ and ASK1, described there, are important hubs of TNF- $\alpha-$ mediated signaling. Their inhibition by miR-199a-5p accounts for the negative feedback activity exerted by it in NF- $\kappa \mathrm{B}$ signaling pathways (Figure 10).

A striking feature of TNF- and other disease-promoting signaling is the induction of miRNAs that initiate a selfsustaining feedback loop to promote disease progression (like miR-21-5p and miR-146b-5p described herein) while suppressing miRNAs that target and inhibit the important effector molecules, attenuating the signaling. ${ }^{59}$ Similar to the results presented herein using cell-based models, the down-regulated miRNAs in BOO controlled many important hubs of signaling, and their dysregulation might account for the phenotypic changes in the bladder. The mechanism of TNF- $\alpha-$ mediated down-regulation of 
miR-199a-5p awaits its investigation. Counteracting its decrease might produce therapeutic benefits, increasing the $\mathrm{SM}$ contractility and reducing the disease-related NF- $\mathrm{KB}$ signaling. Previously, TNF- $\alpha$ has been implicated in developing bladder dysfunction in an animal model of type 2 diabetes, which is associated with bladder hypertrophy and overactivity. ${ }^{60}$ The clinical relevance of our findings is further strengthened by a recent observation that the use of TNF- $\alpha$ antagonist therapies in elderly patients with an autoimmune disease significantly reduces BOO symptoms. ${ }^{61}$ Our results confirm an important role of TNF- $\alpha$ in the regulation of BOO-specific miRNAs and identify miRNAs linking TNF- $\alpha$ signaling and fibrosis. Modulation of expression levels of TNF- $\alpha$-inhibited miRNAs might lead to novel therapeutic approaches for BOO-induced LUTD.

\section{Acknowledgment}

We thank Cornelia Schlup for expert technical assistance.

\section{Supplemental Data}

Supplemental material for this article can be found at https://doi.org/10.1016/j.ajpath.2018.05.008.

\section{References}

1. McVary KT: BPH: epidemiology and comorbidities. Am J Manag Care 2006, 12:S122-S128

2. Koeck I, Burkhard FC, Monastyrskaya K: Activation of common signaling pathways during remodeling of the heart and the bladder. Biochem Pharmacol 2016, 102:7-19

3. Hakenberg OW, Linne C, Manseck A, Wirth MP: Bladder wall thickness in normal adults and men with mild lower urinary tract symptoms and benign prostatic enlargement. Neurourol Urodyn 2000, 19:585-593

4. Kojima M, Inui E, Ochiai A, Naya Y, Ukimura O, Watanabe H: Noninvasive quantitative estimation of infravesical obstruction using ultrasonic measurement of bladder weight. J Urol 1997, 157:476-479

5. Mirone V, Imbimbo C, Longo N, Fusco F: The detrusor muscle: an innocent victim of bladder outlet obstruction. Eur Urol 2007, 51:57-66

6. Metcalfe PD, Wang J, Jiao H, Huang Y, Hori K, Moore RB, Tredget EE: Bladder outlet obstruction: progression from inflammation to fibrosis. BJU Int 2010, 106:1686-1694

7. Gheinani AH, Kiss B, Moltzahn F, Keller I, Bruggmann R, Rehrauer H, Fournier CA, Burkhard FC, Monastyrskaya K: Characterization of miRNA-regulated networks, hubs of signaling, and biomarkers in obstruction-induced bladder dysfunction. JCI Insight 2017, 2:e89560

8. Kusakabe R, Inoue K: Developmental regulation and evolution of muscle-specific microRNAs. Semin Cell Dev Biol 2015, 47-48:9-16

9. Ardekani AM, Naeini MM: The role of microRNAs in human diseases. Avicenna J Med Biotechnol 2010, 2:161-179

10. Gheinani AH, Burkhard FC, Monastyrskaya K: Deciphering microRNA code in pain and inflammation: lessons from bladder pain syndrome. Cell Mol Life Sci 2013, 70:3773-3789

11. Monastyrskaya K, Sanchez-Freire V, Gheinani AH, Klumpp DJ, Babiychuk EB, Draeger A, Burkhard FC: miR-199a-5p regulates urothelial permeability and may play a role in bladder pain syndrome. Am J Pathol 2013, 182:431-448
12. Duan LJ, Qi J, Kong XJ, Huang T, Qian XQ, Xu D, Liang JH, Kang J: miR-133 modulates TGF-beta 1-induced bladder smooth muscle cell hypertrophic and fibrotic response: implication for a role of microRNA in bladder wall remodeling caused by bladder outlet obstruction. Cell Signal 2015, 27:215-227

13. Ekman M, Bhattachariya A, Dahan D, Uvelius B, Albinsson S, Sward K: Mir-29 repression in bladder outlet obstruction contributes to matrix remodeling and altered stiffness. PLoS One 2013, 8:e82308

14. Sadegh MK, Ekman M, Krawczyk K, Svensson D, Goransson O, Dahan D, Nilsson BO, Albinsson S, Uvelius B, Sward K: Detrusor induction of miR-132/212 following bladder outlet obstruction: association with MeCP2 repression and cell viability. PLoS One 2015, 10:e0116784

15. Sanchez Freire V, Burkhard FC, Kessler TM, Kuhn A, Draeger A, Monastyrskaya K: MicroRNAs may mediate the down-regulation of neurokinin-1 receptor in chronic bladder pain syndrome. Am J Pathol 2010, 176:288-303

16. Hashemi Gheinani A, Burkhard FC, Rehrauer H, Aquino Fournier C, Monastyrskaya K: MicroRNA MiR-199a-5p regulates smooth muscle cell proliferation and morphology by targeting WNT2 signaling pathway. J Biol Chem 2015, 290:7067-7086

17. Zhang S, Lv JW, Yang P, Yu Q, Pang J, Wang Z, Guo H, Liu S, Hu J, Li J, Leng J, Huang Y, Ye Z, Wang CY: Loss of dicer exacerbates cyclophosphamide-induced bladder overactivity by enhancing purinergic signaling. Am J Pathol 2012, 181:937-946

18. Perkins ND: Integrating cell-signalling pathways with NF-kappaB and IKK function. Nat Rev Mol Cell Biol 2007, 8:49-62

19. Ma X, Becker Buscaglia LE, Barker JR, Li Y: MicroRNAs in NFkappaB signaling. J Mol Cell Biol 2011, 3:159-166

20. Wiafe B, Adesida A, Churchill T, Adewuyi EE, Li Z, Metcalfe P: Hypoxiaincreased expression of genes involved in inflammation, dedifferentiation, pro-fibrosis, and extracellular matrix remodeling of human bladder smooth muscle cells. In Vitro Cell Dev Biol Anim 2016, 53:58-66

21. Schroder A, Chichester P, Kogan BA, Longhurst PA, Lieb J, Das AK, Levin RM: Effect of chronic bladder outlet obstruction on blood flow of the rabbit bladder. J Urol 2001, 165:640-646

22. Wilson AA, Kwok LW, Porter EL, Payne JG, McElroy GS, Ohle SJ, Greenhill SR, Blahna MT, Yamamoto K, Jean JC, Mizgerd JP, Kotton DN: Lentiviral delivery of RNAi for in vivo lineage-specific modulation of gene expression in mouse lung macrophages. Mol Ther 2013, 21:825-833

23. Bardou P, Mariette J, Escudie F, Djemiel C, Klopp C: jvenn: An interactive Venn diagram viewer. BMC Bioinformatics 2014, 15: 293

24. Sandoval J, Pereda J, Perez S, Finamor I, Vallet-Sanchez A, Rodriguez JL, Franco L: Epigenetic regulation of early- and lateresponse genes in acute pancreatitis. J Immunol 2016, 197:4137-4150

25. Frias B, Lopes T, Pinto R, Cruz F, Cruz CD: Neurotrophins in the lower urinary tract: becoming of age. Curr Neuropharmacol 2011, 9: $553-558$

26. Burger ML, Xue L, Sun Y, Kang C, Winoto A: Premalignant PTENdeficient thymocytes activate microRNAs miR-146a and miR-146b as a cellular defense against malignant transformation. Blood 2014, 123 : 4089-4100

27. Genemaras AA, Ennis H, Kaplan L, Huang CY: Inflammatory cytokines induce specific time- and concentration-dependent MicroRNA release by chondrocytes, synoviocytes, and meniscus cells. J Orthop Res 2016, 34:779-790

28. Oeckinghaus A, Hayden MS, Ghosh S: Crosstalk in NF-kappaB signaling pathways. Nat Immunol 2011, 12:695-708

29. Sethu S, Melendez AJ: New developments on the TNFalphamediated signalling pathways. Biosci Rep 2011, 31:63-76

30. Khan MA: Inflammation signals airway smooth muscle cell proliferation in asthma pathogenesis. Multidiscip Respir Med 2013, 8:11

31. Mullokandov G, Baccarini A, Ruzo A, Jayaprakash AD, Tung N, Israelow B, Evans MJ, Sachidanandam R, Brown BD: Highthroughput assessment of microRNA activity and function using microRNA sensor and decoy libraries. Nat Methods 2012, 9:840-846 
32. Shukla S, Shankar E, Fu P, MacLennan GT, Gupta S: Suppression of NF-kappaB and NF-kappaB-regulated gene expression by apigenin through IkappaBalpha and IKK pathway in TRAMP mice. PLoS One 2015, 10:e0138710

33. Mieczkowski J, Kocyk M, Nauman P, Gabrusiewicz K, Sielska M, Przanowski P, Maleszewska M, Rajan WD, Pszczolkowska D, Tykocki T, Grajkowska W, Kotulska K, Roszkowski M, Kostkiewicz B, Kaminska B: Down-regulation of IKKbeta expression in glioma-infiltrating microglia/macrophages is associated with defective inflammatory/immune gene responses in glioblastoma. Oncotarget 2015, 6:33077-33090

34. Chen R, Alvero AB, Silasi DA, Kelly MG, Fest S, Visintin I, Leiser A, Schwartz PE, Rutherford T, Mor G: Regulation of IKKbeta by miR-199a affects NF-kappaB activity in ovarian cancer cells. Oncogene 2008, 27:4712-4723

35. Dai L, Gu L, Di W: MiR-199a attenuates endometrial stromal cell invasiveness through suppression of the IKKbeta/NF-kappaB pathway and reduced interleukin-8 expression. Mol Hum Reprod 2012, 18:136-145

36. Fujioka S, Niu J, Schmidt C, Sclabas GM, Peng B, Uwagawa T, Li Z, Evans DB, Abbruzzese JL, Chiao PJ: NF-kappaB and AP-1 connection: mechanism of NF-kappaB-dependent regulation of AP1 activity. Mol Cell Biol 2004, 24:7806-7819

37. Karoor V, Fini MA, Loomis Z, Sullivan T, Hersh LB, Gerasimovskaya E, Irwin D, Dempsey EC: Sustained activation of rho GTPases promotes a synthetic pulmonary artery smooth muscle cell phenotype in neprilysin null mice. Arterioscler Thromb Vasc Biol 2018, 38:154-163

38. Bielenberg DR, Seth A, Shimizu A, Pelton K, Cristofaro V, Ramachandran A, Zwaans BM, Chen C, Krishnan R, Seth M, Huang L, Takashima S, Klagsbrun M, Sullivan MP, Adam RM: Increased smooth muscle contractility in mice deficient for neuropilin 2. Am J Pathol 2012, 181:548-559

39. Vasquez E, Cristofaro V, Lukianov S, Burkhard FC, Gheinani AH, Monastyrskaya K, Bielenberg DR, Sullivan MP, Adam RM: Deletion of neuropilin 2 enhances detrusor contractility following bladder outlet obstruction. JCI Insight 2017, 2:e90617

40. Djavan B, Fong YK, Harik M, Milani S, Reissigl A, Chaudry A, Anagnostou T, Bagheri F, Waldert M, Kreuzer S, Fajkovic H, Marberger M: Longitudinal study of men with mild symptoms of bladder outlet obstruction treated with watchful waiting for four years. Urology 2004, 64:1144-1148

41. Thomas AW, Cannon A, Bartlett E, Ellis-Jones J, Abrams P: The natural history of lower urinary tract dysfunction in men: minimum 10-year urodynamic follow-up of untreated bladder outlet obstruction. BJU Int 2005, 96:1301-1306

42. Bechis SK, Otsetov AG, Ge R, Olumi AF: Personalized medicine for the management of benign prostatic hyperplasia. J Urol 2014, 192:16-23

43. Chancellor MB: The overactive bladder progression to underactive bladder hypothesis. Int Urol Nephrol 2014, 46 Suppl 1:S23-S27

44. Lehrke M, Lazar MA: The many faces of PPARgamma. Cell 2005, 123:993-999

45. Kanefsky J, Lenburg M, Hai CM: Cholinergic receptor and cyclic stretch-mediated inflammatory gene expression in intact ASM. Am J Respir Cell Mol Biol 2006, 34:417-425

46. Gwyer Findlay E, Hussell T: Macrophage-mediated inflammation and disease: a focus on the lung. Mediators Inflamm 2012, 2012: 140937
47. Awad AS, You H, Gao T, Cooper TK, Nedospasov SA, Vacher J, Wilkinson PF, Farrell FX, Brian Reeves W: Macrophage-derived tumor necrosis factor-alpha mediates diabetic renal injury. Kidney Int 2015, 88:722-733

48. Adegunsoye A, Balachandran J: Inflammatory response mechanisms exacerbating hypoxemia in coexistent pulmonary fibrosis and sleep apnea. Mediators Inflamm 2015, 2015:510105

49. Liang Z, Xin W, Qiang L, Xiang C, Bang-Hua L, Jin Y, De-Yi L, Hong L, Kun-Jie W: Hydrostatic pressure and muscarinic receptors are involved in the release of inflammatory cytokines in human bladder smooth muscle cells. Neurourol Urodyn 2017, 36:1261-1269

50. Mace TA, Collins AL, Wojcik SE, Croce CM, Lesinski GB, Bloomston M: Hypoxia induces the overexpression of microRNA-21 in pancreatic cancer cells. J Surg Res 2013, 184:855-860

51. Mendell JT, Olson EN: MicroRNAs in stress signaling and human disease. Cell 2012, 148:1172-1187

52. Iliopoulos D, Jaeger SA, Hirsch HA, Bulyk ML, Struhl K: STAT3 activation of miR-21 and miR-181b-1 via PTEN and CYLD are part of the epigenetic switch linking inflammation to cancer. Mol Cell 2010, 39:493-506

53. Chen J, Shin VY, Siu MT, Ho JC, Cheuk I, Kwong A: miR-199a-5p confers tumor-suppressive role in triple-negative breast cancer. BMC Cancer 2016, 16:887

54. Hayakawa R, Hayakawa T, Takeda K, Ichijo H: Therapeutic targets in the ASK1-dependent stress signaling pathways. Proc Jpn Acad Ser B Phys Biol Sci 2012, 88:434-453

55. Goebeler M, Gillitzer R, Kilian K, Utzel K, Brocker EB, Rapp UR, Ludwig S: Multiple signaling pathways regulate NF-kappaBdependent transcription of the monocyte chemoattractant protein-1 gene in primary endothelial cells. Blood 2001, 97:46-55

56. Misra UK, Deedwania R, Pizzo SV: Activation and cross-talk between Akt, NF-kappaB, and unfolded protein response signaling in 1$\mathrm{LN}$ prostate cancer cells consequent to ligation of cell surfaceassociated GRP78. J Biol Chem 2006, 281:13694-13707

57. Beyaert R, Cuenda A, Vanden Berghe W, Plaisance S, Lee JC, Haegeman G, Cohen P, Fiers W: The p38/RK mitogen-activated protein kinase pathway regulates interleukin- 6 synthesis response to tumor necrosis factor. EMBO J 1996, 15:1914-1923

58. Mnich SJ, Blanner PM, Hu LG, Shaffer AF, Happa FA, O'Neil S, Ukairo O, Weiss D, Welsh E, Storer C, Mbalaviele G, Ichijo H, Monahan JB, Hardy MM, Eda H: Critical role for apoptosis signalregulating kinase 1 in the development of inflammatory $\mathrm{K} / \mathrm{BxN}$ serum-induced arthritis. Int Immunopharmacol 2010, 10:1170-1176

59. Fiorillo AA, Heier CR, Novak JS, Tully CB, Brown KJ, Uaesoontrachoon K, Vila MC, Ngheim PP, Bello L, Kornegay JN, Angelini C, Partridge TA, Nagaraju K, Hoffman EP: TNF-alphainduced microRNAs control dystrophin expression in becker muscular dystrophy. Cell Rep 2015, 12:1678-1690

60. Wang Z, Cheng Z, Cristofaro V, Li J, Xiao X, Gomez P, Ge R, Gong E, Strle K, Sullivan MP, Adam RM, White MF, Olumi AF: Inhibition of TNF-alpha improves the bladder dysfunction that is associated with type 2 diabetes. Diabetes 2012, 61:2134-2145

61. Lapan B, Franco O, Pruitt J, Petkewicz J, Helfand B, Brendler C, Wang C, Hayward S: TNFa antagonists reduce incidence of BPH in patients with autoimmune inflammatory conditions. J Urol 2017, 197 : e212 